\title{
Global burden of disease due to smokeless tobacco consumption in adults: analysis of data from 113 countries
}

\author{
Kamran Siddiqi ${ }^{1 *}$, Sarwat Shah ${ }^{1}$, Syed Muslim Abbas², Aishwarya Vidyasagaran ${ }^{1}$, Mohammed Jawad ${ }^{3}$,
} Omara Dogar ${ }^{1}$ and Aziz Sheikh ${ }^{4}$

\begin{abstract}
Background: Smokeless tobacco is consumed in most countries in the world. In view of its widespread use and increasing awareness of the associated risks, there is a need for a detailed assessment of its impact on health. We present the first global estimates of the burden of disease due to consumption of smokeless tobacco by adults.

Methods: The burden attributable to smokeless tobacco use in adults was estimated as a proportion of the disability-adjusted life-years (DALYs) lost and deaths reported in the 2010 Global Burden of Disease study. We used the comparative risk assessment method, which evaluates changes in population health that result from modifying a population's exposure to a risk factor. Population exposure was extrapolated from country-specific prevalence of smokeless tobacco consumption, and changes in population health were estimated using disease-specific risk estimates (relative risks/odds ratios) associated with it. Country-specific prevalence estimates were obtained through systematically searching for all relevant studies. Disease-specific risks were estimated by conducting systematic reviews and meta-analyses based on epidemiological studies.
\end{abstract}

Results: We found adult smokeless tobacco consumption figures for 115 countries and estimated burden of disease figures for 113 of these countries. Our estimates indicate that in 2010, smokeless tobacco use led to 1.7 million DALYs lost and 62,283 deaths due to cancers of mouth, pharynx and oesophagus and, based on data from the benchmark 52 country INTERHEART study, 4.7 million DALYs lost and 204,309 deaths from ischaemic heart disease. Over $85 \%$ of this burden was in South-East Asia.

Conclusions: Smokeless tobacco results in considerable, potentially preventable, global morbidity and mortality from cancer; estimates in relation to ischaemic heart disease need to be interpreted with more caution, but nonetheless suggest that the likely burden of disease is also substantial. The World Health Organization needs to consider incorporating regulation of smokeless tobacco into its Framework Convention for Tobacco Control.

\section{Background}

Smokeless tobacco (SLT) consists of a number of products containing tobacco, which are consumed-without burning-through the mouth or nose [1]. A diverse range of SLT products are available worldwide, varying in their composition, methods of preparation and consumption, and associated health risks (Table 1) [1]. Its use is most prevalent in South and South-East Asia where one-third of tobacco is consumed in smokeless

\footnotetext{
* Correspondence: Kamran.siddiqi@york.ac.uk

${ }^{1}$ Department of Health Sciences, Hull York Medical School, University of York, Room 105a, First floor, ARRC Building, Heslington, York YO10 5DD, UK Full list of author information is available at the end of the article
}

form [2, 3]. Wrapped in a betel leaf with areca nut, slaked lime, and catechu, SLT is often served at social occasions in this region. Other products (e.g. gutkha, khaini) contain slaked lime, areca nut, flavourings, and aromatic substances [4]. A number of products based on powdered tobacco (e.g. snus) are also consumed in Nordic countries and North America. In other parts of world, the most commonly used SLT products (Table 1) include Chimó (Venezuela), Nass (Uzbekistan, Kyrgyzstan), Tambook (Sudan, Chad), and Snuff (Nigeria, Ghana, South Africa).

In addition to nicotine, SLT products contain over 30 carcinogens [5] including tobacco-specific nitrosamines 
Table 1 Smokeless tobacco products consumed most commonly across the world

\begin{tabular}{|c|c|c|c|c|c|c|c|}
\hline $\begin{array}{l}\text { Smokeless tobacco } \\
\text { products }\end{array}$ & Regions (WHO) & Countries (highest consumption) & Other ingredients & Preparation and use & $\mathrm{pH}^{\mathrm{a}}$ & Nicotine $^{a}(\mathrm{mg} / \mathrm{g})$ & Total TSNA $(\mathrm{ng} / \mathrm{g})$ \\
\hline Snus (Swedish) & Europe (Region A) & $\begin{array}{l}\text { Nordic countries (Denmark, } \\
\text { Finland, Iceland, Norway, } \\
\text { Sweden) }\end{array}$ & $\begin{array}{l}\text { Water, sodium carbonate, } \\
\text { sodium chloride, moisturisers, } \\
\text { flavouring }\end{array}$ & $\begin{array}{l}\text { A heat treatment process; } \\
\text { placed between the gum } \\
\text { and upper lip }\end{array}$ & $6.6-7.2$ & $7.8-15.2$ & $601-723$ \\
\hline \multirow{4}{*}{$\begin{array}{l}\text { Plug, Snuff (US), } \\
\text { Snus (US) }\end{array}$} & \multirow[t]{4}{*}{ Americas (Region A and B) } & \multirow[t]{4}{*}{ US, Canada, Mexico } & \multirow[t]{4}{*}{ Sweeteners, liquorice } & Plug; air cured & \multirow[t]{4}{*}{$4.7-7.8$} & \multirow[t]{4}{*}{$3.9-40.1$} & \multirow[t]{4}{*}{$313-76,500$} \\
\hline & & & & $\begin{array}{l}\text { Dry or moist snuff; finely } \\
\text { ground and fire cured }\end{array}$ & & & \\
\hline & & & & Snus; steam cured & & & \\
\hline & & & & $\begin{array}{l}\text { Snuff; kept between lip and } \\
\text { gum, dry snuff can be } \\
\text { inhaled too }\end{array}$ & & & \\
\hline Chimó & Americas (Region B) & Venezuela, Colombia & $\begin{array}{l}\text { Sodium bicarbonate, brown } \\
\text { sugar, Mamo'n tree ashes }\end{array}$ & $\begin{array}{l}\text { Tobacco paste made from } \\
\text { tobacco leaves; placed between } \\
\text { the lip or cheek and gum and } \\
\text { left there for some time }\end{array}$ & $6.9-9.4$ & $5.3-30.1$ & 9390 \\
\hline Nass (Naswar) & $\begin{array}{l}\text { Europe (Region B) and Eastern } \\
\text { Mediterranean (Region D) }\end{array}$ & $\begin{array}{l}\text { Uzbekistan, Kyrgyzstan, Tajikistan, } \\
\text { Afghanistan, Pakistan, Iran }\end{array}$ & $\begin{array}{l}\text { Lime, ash, flavourings } \\
\text { (cardamom), indigo }\end{array}$ & $\begin{array}{l}\text { Sundried and powdered; placed } \\
\text { between lip or cheek and gum }\end{array}$ & $8.4-9.1$ & $8.9-14.2$ & $478-1380$ \\
\hline Tambook & $\begin{array}{l}\text { Eastern Mediterranean (Region D) } \\
\text { and Africa (Region D) }\end{array}$ & Sudan, Chad & $\begin{array}{l}\text { Mixed with moist sodium } \\
\text { bicarbonate }\end{array}$ & $\begin{array}{l}\text { Fermented and grounded; } \\
\text { placed and kept in mouth }\end{array}$ & $7.3-10.1$ & $9.6-28.2$ & $302,000-992,000$ \\
\hline \multirow[t]{2}{*}{$\begin{array}{l}\text { Snuff (North and } \\
\text { West African) }\end{array}$} & \multirow[t]{2}{*}{ Africa (Region D) } & \multirow[t]{2}{*}{$\begin{array}{l}\text { Nigeria, Ghana, Algeria, } \\
\text { Cameroon, Chad, Senegal }\end{array}$} & \multirow{2}{*}{$\begin{array}{l}\text { Dried tobacco leaves mixed } \\
\text { with potassium nitrate and } \\
\text { other salts }\end{array}$} & $\begin{array}{l}\text { Dry snuff; finely ground and } \\
\text { inhaled as a pinch }\end{array}$ & \multirow[t]{2}{*}{$9.0-9.4$} & \multirow[t]{2}{*}{$2.5-7.4$} & \multirow[t]{2}{*}{$1520-2420$} \\
\hline & & & & Moist snuff is placed in mouth & & & \\
\hline Snuff (South African) & Africa (Region E) & South Africa & $\begin{array}{l}\text { Dried tobacco leaves mixed } \\
\text { with ash }\end{array}$ & $\begin{array}{l}\text { Dry snuff; finely ground and } \\
\text { inhaled as a pinch }\end{array}$ & $6.5-10.1$ & $1.2-17.2$ & $1710-20,500$ \\
\hline Khaini & \multirow{3}{*}{$\begin{array}{l}\text { South East Asia (Regions B and D) } \\
\text { Western Pacific (Region B) } \\
\text { Eastern Mediterranean (Region D) } \\
\text { Europe (Region A) }\end{array}$} & India, Bangladesh, Nepal, Bhutan & $\begin{array}{l}\text { Slaked lime, menthol, } \\
\text { flavourings, areca nut }\end{array}$ & $\begin{array}{l}\text { Shredded; kept in mouth } \\
\text { between lips and gum }\end{array}$ & $9.6-9.8$ & $2.5-4.8$ & $21,600-23,900$ \\
\hline Zarda & & $\begin{array}{l}\text { Bangladesh, India, Pakistan, } \\
\text { Myanmar, Thailand, Indonesia, } \\
\text { Nepal, Maldives, Sri Lanka, UK }\end{array}$ & $\begin{array}{l}\text { Served wrapped in a betel } \\
\text { leaf with lime, catechu, } \\
\text { areca nuts }\end{array}$ & $\begin{array}{l}\text { Shredded tobacco leaves are } \\
\text { boiled with lime and saffron; } \\
\text { the mixture is dried then } \\
\text { chewed and spat }\end{array}$ & $5.2-6.5$ & $9.5-30.4$ & $5490-53,700$ \\
\hline Gutkha & & $\begin{array}{l}\text { India, Pakistan, Bangladesh, } \\
\text { Nepal, Myanmar, Sri Lanka, UK }\end{array}$ & $\begin{array}{l}\text { Betel nut, catechu, flavourings, } \\
\text { sweeteners }\end{array}$ & $\begin{array}{l}\text { Commercially manufactured; } \\
\text { sucked, chewed, and spat }\end{array}$ & $7.4-8.9$ & $0.2-4.2$ & $83-23,900$ \\
\hline
\end{tabular}


(TSNA), arsenic, beryllium, cadmium, nickel, chromium, nitrite, and nitrate. The level of nicotine and carcinogens vary between products (Table 1) [6]. For example, nicotine content among SLT products varies between 0.2 and $40.1 \mathrm{mg} / \mathrm{g}$, compared to commercial filtered cigarettes which contain $16.3 \mathrm{mg} / \mathrm{g}$ of nicotine [7]. Their $\mathrm{pH}$ also varies, which, being a key determinant of the level of absorption of nicotine and carcinogens, determines its toxicity: the higher the $\mathrm{pH}$, the higher the absorption and, consequently, the higher the toxicity [6]. Such considerations mean that there are substantial variations between different SLT products in the level of risk posed to human health $[4,8-11]$. It is therefore important not to consider SLT as a single product, but rather as groups of products with differences in their toxicity and addictiveness depending upon their carcinogen, nicotine, and $\mathrm{pH}$ levels. The diversity in SLT toxicity has been an impediment not only in establishing its global risks to human health, but also in agreeing on international policies for its prevention and control. It is therefore perhaps unsurprising that despite several country-specific studies [12-15] no attempt has hitherto been made to estimate its global disease burden.

To overcome these challenges, we developed a novel approach to estimate the global burden associated with the use of SLT products. The determinants of their toxicity (carcinogens and $\mathrm{pH}$ ) and addictiveness (nicotine) are dependent on preparation methods, ingredients that are added to SLT products, and consumption behaviours. Given that the SLT preparations and consumption patterns are determined by, and vary with, geography and culture [16], it is possible to group them according to their availability in different parts of the world (Table 1). These groups of SLT products, classified according to different geographical regions, will also be distinguishable from each other on the basis of their toxicity, addictiveness, and associated health risks. Hence, the risks were assumed to be highest in those regions and cultures where products are combined with other ingredients, and are prepared and consumed in a way that makes them very alkaline (i.e. a high $\mathrm{pH}$ ), and rich in nicotine and TSNA [6, 17]. Building on this assumption, we aimed to estimate the worldwide burden of disease attributable to SLT use, measured in terms of disability adjusted life years (DALYs) lost and number of deaths in 2010.

\section{Methods}

We used the comparative risk assessment method, which evaluates changes in population health (burden of disease) that result from modifying a population's exposure to a risk factor $[18,19]$. For this, we used 2010 datasets, which provided the most recent global estimates of burden of disease [20]. The estimates were calculated for individual countries and then grouped into 14 World
Health Organization (WHO) sub-regions (Additional file 1: Appendix 1) [21]. These were generated through estimating the following:

1. The prevalence of SLT consumption

2. Diseases caused by SLT use

3. The relative risks of acquiring these diseases

4. The population attributable fraction (PAF) for each of these diseases

5. The overall burden of these diseases in terms of DALYs lost and deaths

6. Proportion of this burden attributable to SLT use

\section{Prevalence of smokeless tobacco use}

We carried out a systematic literature search (see Additional file 1: Appendix 2 for a detailed description of the methods employed) for the point prevalence (current use) of SLT consumption among all adult ( $\geq 15$ years) populations, and also for men and women separately. Only one prevalence report was included for one country. Latest national prevalence data collected as part of an international or regional survey were preferred over an older isolated national or a sub-national survey. We used data from the Global Adult Tobacco Survey (GATS), where available [22]. In its absence, other international (WHO STEPwise approach to Surveillance, The Demographic and Health Surveys), regional (Special Europe Barometer), national, and/or sub-national surveys were used to extract prevalence data.

\section{Diseases caused by smokeless tobacco use}

A scoping review was carried out to identify associated diseases. A series of focused literature reviews were subsequently carried out to find and assess the evidence of causation between each of these diseases and SLT use. Our search strategies and selection criteria are provided in Additional file 1: Appendix 3. One researcher ran the searches, which were then independently scrutinised by another independent researcher who considered the search results against the pre-specified inclusion and exclusion criteria. Similarly, one researcher extracted data, which were independently crosschecked by another researcher. In particular, we appraised the studies for case definitions for diseases and for assessment methods for measuring exposure to SLT and for investigating the effects of potential confounders. We excluded those diseases (and respective studies) where evidence was not supportive of a causal relationship. Only studies that adequately controlled for smoking and/or alcohol as potential confounders either at the design or the analysis stage were carried forward into the next stage of the analysis (discussed below). Quality was assessed using the Newcastle-Ottawa Scale for assessing the quality of nonrandomised studies in meta-analyses [23]. 


\section{Assessing risk and meta-analyses}

Risk estimates (relative risks/odds ratios) and their confidence intervals $(\mathrm{CI})$ were log transformed to produce effect sizes and standard errors, respectively [24]. We carried out random effects meta-analysis using RevMan version 5 to estimate pooled risk estimates. We first obtained country-specific risk estimates (relative risks/odds ratios) for individual diseases by pooling data from the included studies carried out in respective countries. We then extrapolated non-specific global risk estimates by pooling respective country-specific risk estimates. We were mindful that the risk of acquiring diseases varies between countries owing to differences in SLT products used. Therefore, for each disease where good countryspecific risk estimates (pooled estimate from a metaanalysis of three or more studies in respective country) were available, we applied these to respective countries and also to those countries and regions where similar SLT products are used. In the absence of good countryspecific risk estimates, we used either one of the following two approaches: (a) In countries and regions that use SLT products with moderate to high $\mathrm{pH}$ and TSNAs levels, we applied non-specific global estimates (pooled estimate from a meta-analysis of all studies); and (b) in countries and regions where there was either no information available on the SLT products or the information available indicates low levels of $\mathrm{pH}$ and TSNA, we did not apply any estimates. Further details on the application of these assumptions across all 14 WHO regions are provided in web Additional file 1: Appendix 4. We only used those pooled relative risks (country or nonspecific) that were found to be statistically significant.

Where associations were presented for more than one SLT product in the same paper, we considered these as separate studies for the purpose of meta-analysis. Similarly, where risks were given separately for former and current SLT users, these were also treated as separate studies. We did not attempt to group risks according to gender because very few studies had such sub-group analysis.

\section{Population attributable fraction}

PAF is the proportional reduction in disease or mortality that would occur if exposure were reduced to zero $[25,26]$. PAF was estimated for each disease for each country for both males and females, using the following formula:

$$
\begin{aligned}
& \mathrm{PAF}=\mathrm{P}_{\mathrm{e}}\left(\mathrm{RR}_{\mathrm{e}}-1\right) /\left[1+\mathrm{P}_{\mathrm{e}}\left(\mathrm{RR}_{\mathrm{e}}-1\right)\right] \\
& \mathrm{P}_{\mathrm{e}}=\text { Prevalence } \\
& \mathrm{RR}_{\mathrm{e}}=\text { Relative Risk }
\end{aligned}
$$

\section{Overall burden}

The overall number of DALYs and deaths for each associated disease for both males and females for each country were extracted from the 2010 Global Burden of Disease study [27, 28].

\section{Attributable burden}

The attributable burden (AB), in deaths and DALYs, was estimated for each associated disease for each country for both males and females by multiplying PAF by the overall burden of the disease (B):

$$
\mathrm{AB}=\mathrm{PAF} \times \mathrm{B}
$$

\section{Results}

\section{Prevalence of smokeless tobacco use}

We found adult prevalence figures for SLT consumption in 115 countries (Fig. 1). The definition for 'adult' ranged from 15, 16, 25, or 35 years at one end to $49,64,65,70$, $74,84,85,89$, or no age limit at the other. The PRISMA diagram describing the selection of the prevalence reports is provided in Additional file 1: Appendix 5a.

In general, SLT consumption was higher among males than females (Table 2). Mauritania had the highest prevalence of SLT consumption among females (28.3\%), followed by Bangladesh (27.9 \%), Madagascar (19.6\%), India (18.4 \%), and Bhutan (17.3\%). Among males, Myanmar (51.4 \%), Nepal (37.9 \%), India (32.9 \%), Uzbekistan (31.8 \%), and Bangladesh (26.4 \%) had the highest consumption rates. Within Europe, SLT (snus) consumption was high in Sweden $(24.0 \%$ males, $7.0 \%$ females) and Norway (20.0 \% males, $6.0 \%$ females).

\section{Diseases caused by smokeless tobacco use}

The initial scoping review identified a number of associated diseases, including a range of cancers, cardiovascular diseases (ischaemic heart disease and stroke), periodontal conditions, and adverse pregnancy outcomes. The subsequent more focused systematic reviews identified 53 studies (Table 3) reporting association between SLT consumption and cancers of mouth, pharynx, larynx, oesophagus, lung, and pancreas (39 studies); and cardiovascular diseases, such as ischaemic heart disease and stroke (14 studies). PRISMA flow diagrams describing the selection process of the studies identified in the literature searches are provided in Additional file 1: Appendix 5b,c. The pooled non-specific relative risks were statistically significant for cancers of the mouth, pharynx, and oesophagus (Figs. 2, 3, 4, and 5). Only statistically significant relative risks (country-specific or non-specific) were included in the model to estimate attributable risks. For example, the pooled nonspecific relative risk for laryngeal cancer was $1.42(95 \% \mathrm{CI}$ 0.77-2.59), and hence excluded (Additional file 1 : Appendix 6). Likewise, none of the country-specific estimates for the USA were statistically significant 


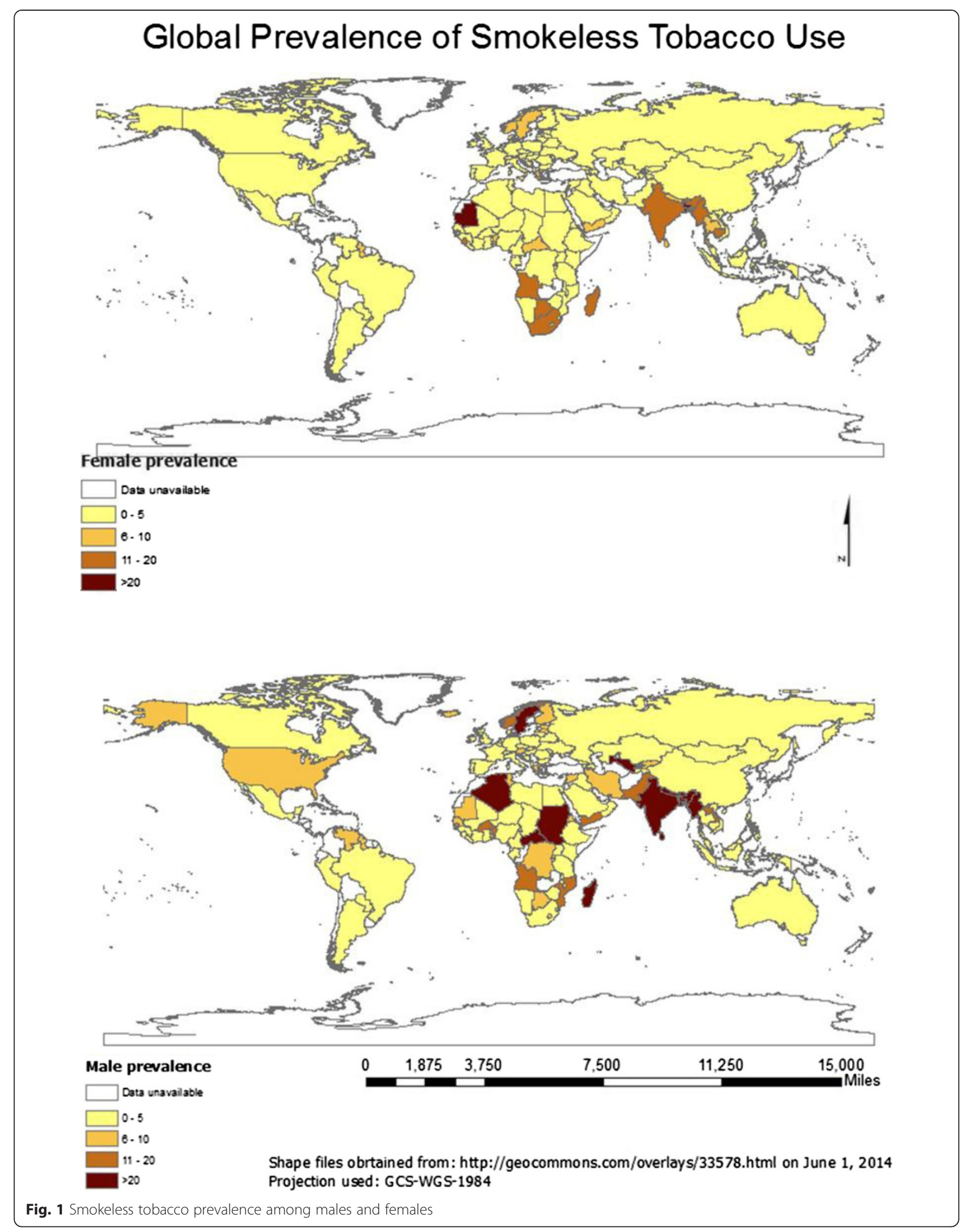


Table 2 Prevalence of smokeless tobacco use in different countries of the world according to WHO sub-regional classification

\begin{tabular}{|c|c|c|c|c|c|}
\hline WHO sub-regions & Country & M & $\mathrm{F}$ & Source & Year \\
\hline \multirow[t]{21}{*}{ Africa (Region D) } & Algeria & 21 & 0.4 & STEPS [38] & 2005 \\
\hline & Benin & 12.7 & 5.7 & STEPS [38] & 2008 \\
\hline & Burkina Faso & - & 3.86 & DHS [39] & 2011 \\
\hline & Cameroon & 1.94 & 0.94 & DHS [39] & 2011 \\
\hline & Cape Verde & 3.5 & 5.8 & STEPS [38] & 2007 \\
\hline & Chad & 1.9 & 0.4 & STEPS [38] & 2008 \\
\hline & Comoros & 7.72 & 2.99 & DHS [39] & 2012 \\
\hline & Gabon & 0.48 & 0.34 & DHS [39] & 2012 \\
\hline & Gambia & 0.8 & 1.4 & STEPS [38] & 2010 \\
\hline & Ghana & 1.33 & 0.2 & DHS [39] & 2008 \\
\hline & Guinea & 1.4 & 1.5 & STEPS [38] & 2009 \\
\hline & Liberia & 2.3 & 2.4 & DHS [40] & 2007 \\
\hline & Madagascar & 24.66 & 19.6 & DHS [39] & 2009 \\
\hline & Mali & 5 & 1.2 & STEPS [38] & 2007 \\
\hline & Mauritania & 5.7 & 28.3 & STEPS [38] & 2006 \\
\hline & Niger & 4.55 & 2.3 & DHS [39] & 2012 \\
\hline & Nigeria & 3.2 & 0.5 & DHS [40] & 2008 \\
\hline & Sao Tome \& Principe & 3.8 & 1.9 & STEPS [38] & 2009 \\
\hline & Senegal & 6.63 & 0.23 & DHS [39] & 2011 \\
\hline & Sierra Leone & 3 & 12 & STEPS [38] & 2009 \\
\hline & Togo & 5.1 & 2.2 & STEPS [38] & 2010 \\
\hline \multirow[t]{19}{*}{ Africa (Region E) } & Botswana & 7.2 & 14.5 & STEPS [38] & 2007 \\
\hline & Burundi & 0.03 & 0.31 & DHS [39] & 2011 \\
\hline & Congo (Brazzaville) & 8.3 & 1.54 & DHS [39] & 2012 \\
\hline & Congo (Republic) & 8.67 & 3.22 & DHS [39] & 2013 \\
\hline & Cote d'Ivoire & 0.61 & 1.27 & DHS [39] & 2012 \\
\hline & Eritrea & 5.8 & 0.2 & STEPS [38] & 2004 \\
\hline & Ethiopia & 1.94 & 0.2 & DHS [39] & 2011 \\
\hline & Kenya & 2.05 & 1.29 & DHS [39] & 2008 \\
\hline & Lesotho & 1.3 & 9.1 & DHS [40] & 2009 \\
\hline & Malawi & 1.9 & 5 & STEPS [38] & 2009 \\
\hline & Mozambique & 10.94 & 0.82 & DHS [39] & 2011 \\
\hline & Namibia & 1.8 & 2.3 & DHS [40] & 2006-07 \\
\hline & Rwanda & 5.8 & 2.73 & DHS [39] & 2011 \\
\hline & South Africa & 2.4 & 10.9 & DHS [41] & 2003 \\
\hline & Swaziland & 2.6 & 0.8 & STEPS [38] & 2007 \\
\hline & Tanzania & 2.03 & 0.83 & DHS [39] & 2010 \\
\hline & Uganda & 2.94 & 1.5 & DHS [39] & 2011 \\
\hline & Zambia & 0.3 & 1.2 & DHS [39] & 2007 \\
\hline & Zimbabwe & 1.6 & 0.4 & DHS [41] & 2011 \\
\hline \multirow[t]{2}{*}{ Americas (Region A) } & Canada & 2 & - & ICS [41] & 2011 \\
\hline & USA & 6.5 & 0.4 & ICS [41] & 2010 \\
\hline
\end{tabular}


Table 2 Prevalence of smokeless tobacco use in different countries of the world according to WHO sub-regional classification (Continued)

\begin{tabular}{|c|c|c|c|c|c|}
\hline \multirow[t]{10}{*}{ Americas (Region B) } & Argentina & 0.1 & 0.2 & GATS [42] & 2012 \\
\hline & Barbados & 0 & 0.6 & STEPS [38] & 2007 \\
\hline & Brazil & 0.6 & 0.3 & GATS [42] & 2010 \\
\hline & Dominican Republic & 1.9 & 0.3 & DHS [40] & 2007 \\
\hline & Grenada & 2.2 & 0.3 & STEPS [38] & 2011 \\
\hline & Mexico & 0.3 & 0.3 & GATS [42] & 2009 \\
\hline & Paraguay & 3 & 1.6 & ICS [41] & 2011 \\
\hline & St Kitts \& Nevis ${ }^{a}$ & 0.3 & 0.1 & STEPS [38] & 2007 \\
\hline & Trinidad \& Tobago & 0.5 & 0.3 & STEPS [38] & 2011 \\
\hline & Venezuela & 6.2 & 0.9 & ICS [41] & 2011 \\
\hline Americas (Region D) & Haiti & - & 2.5 & DHS [40] & $2005-06$ \\
\hline \multirow[t]{3}{*}{ Eastern Mediterranean (Region B) } & Libya & 2.2 & 0.1 & STEPS [38] & 2009 \\
\hline & Saudi Arabia & 1.3 & 0.5 & STEPS [38] & 2004 \\
\hline & Tunisia & 8.6 & 2.2 & ICS [41] & $2005-06$ \\
\hline \multirow[t]{5}{*}{ Eastern Mediterranean (Region D) } & Egypt & 4.8 & 0.3 & GATS [42] & 2009 \\
\hline & Iraq & 1.6 & 0.3 & STEPS [38] & 2006 \\
\hline & Pakistan & 16.3 & 2.44 & DHS [43] & $2012-13$ \\
\hline & Sudan & 24.1 & 1 & STEPS [38] & 2005 \\
\hline & Yemen & 15.1 & 6.2 & ICS [41] & 2003 \\
\hline \multirow[t]{21}{*}{ Europe (Region A) } & Austria & 7.8 & 1.1 & SEBS [44] & 2012 \\
\hline & Belgium & 1.1 & 0.6 & SEBS [44] & 2012 \\
\hline & Cyprus & 2.1 & 0.4 & SEBS [44] & 2012 \\
\hline & Czech Republic & 2.5 & 0.4 & SEBS [44] & 2012 \\
\hline & Denmark & 3 & 1 & ICS [41] & 2010 \\
\hline & Finland & 5.5 & 0.3 & ICS [41] & 2011 \\
\hline & France & 1.2 & 0.6 & SEBS [44] & 2012 \\
\hline & Germany & 3.4 & 3.4 & SEBS [44] & 2012 \\
\hline & Iceland & 5.97 & - & ICS [41] & 2008 \\
\hline & Ireland & 2.2 & 0.9 & SEBS [44] & 2012 \\
\hline & Italy & 1.8 & 1.5 & SEBS [44] & 2012 \\
\hline & Luxembourg & 1.8 & 1 & SEBS [44] & 2012 \\
\hline & Malta & 5.5 & 1.5 & SEBS [44] & 2012 \\
\hline & Netherlands & 0.3 & 0.1 & ICS [41] & 2011 \\
\hline & Norway & 20 & 6 & ICS [41] & 2011 \\
\hline & Portugal & 4.4 & 1.1 & SEBS [44] & 2012 \\
\hline & Slovenia & 1.8 & 0.4 & SEBS [44] & 2012 \\
\hline & Spain & 0.4 & 0.2 & SEBS [44] & 2012 \\
\hline & Sweden & 24 & 7 & ICS [41] & 2011 \\
\hline & Switzerland & 4 & 1.3 & ICS [41] & 2011 \\
\hline & United Kingdom & 1.6 & 0.5 & SEBS [44] & 2012 \\
\hline \multirow[t]{4}{*}{ Europe (Region B) } & Ajerbaijan & 0.3 & 0 & DHS [40] & 2006 \\
\hline & Armenia & 1.8 & 0 & DHS [40] & 2005 \\
\hline & Bulgaria & 0.3 & 0 & SEBS [44] & 2012 \\
\hline & Georgia & 1 & 0.2 & ICS [41] & 2010 \\
\hline
\end{tabular}


Table 2 Prevalence of smokeless tobacco use in different countries of the world according to WHO sub-regional classification (Continued)

\begin{tabular}{|c|c|c|c|c|c|}
\hline & Kyrgyzstan & 7 & 0.3 & ICS [41] & 2006 \\
\hline & Poland & 1 & 0.1 & GATS [42] & 2009 \\
\hline & Romania & 0.4 & 0.2 & GATS [42] & 2011 \\
\hline & Slovakia & 3.9 & 0.7 & SEBS [44] & 2012 \\
\hline & Uzbekistan & 31.8 & 0.2 & DHS [40] & 2002 \\
\hline \multirow[t]{5}{*}{ Europe (Region C) } & Latvia & 5.8 & 0.9 & ICS [41] & 2010 \\
\hline & Lithuania & 1.2 & 0.2 & SEBS [44] & 2012 \\
\hline & Moldova & 0.1 & 0 & DHS [40] & 2005 \\
\hline & Russia & 1 & 0.2 & GATS [42] & 2009 \\
\hline & Ukraine & 0.5 & 0 & GATS [42] & 2010 \\
\hline \multirow[t]{3}{*}{ South East Asia (Region B) } & Indonesia & 1.5 & 2 & GATS [42] & 2011 \\
\hline & Sri Lanka & 24.9 & 6.9 & STEPS [38] & 2006 \\
\hline & Thailand & 1.1 & 5.2 & GATS [42] & 2011 \\
\hline \multirow[t]{7}{*}{ South East Asia (Region D) } & Bangladesh & 26.4 & 27.9 & GATS [42] & 2009 \\
\hline & Bhutan & 21.1 & 17.3 & STEPS [38] & 2007 \\
\hline & India & 32.9 & 18.4 & GATS [42] & 2009 \\
\hline & Maldives & 5.6 & 2.6 & STEPS [38] & 2011 \\
\hline & Myanmar & 51.4 & 16.1 & STEPS [38] & 2009 \\
\hline & Nepal & 37.9 & 6 & DHS [41] & 2011 \\
\hline & Timor Leste & 2.48 & 1.93 & DHS [43] & $2009-10$ \\
\hline Western Pacific (Region A) & Australia & 0.75 & 0.41 & ICS [45] & 2004 \\
\hline \multirow[t]{8}{*}{ Western Pacific (Region B) } & Cambodia & 2.2 & 14.8 & STEPS [38] & 2010 \\
\hline & China & 0.7 & 0 & GATS [42] & 2010 \\
\hline & Lao People's Democratic Republic & 14.6 & 1.1 & STEPS [38] & 2008 \\
\hline & Malaysia & 0.9 & 0.6 & GATS [42] & 2011 \\
\hline & Micronesia & 22.4 & 3 & STEPS [38] & 2002 \\
\hline & Mongolia & 2.8 & 0.5 & STEPS [38] & 2009 \\
\hline & Philippines & 2.8 & 1.2 & GATS [42] & 2009 \\
\hline & Vietnam & 0.3 & 2.3 & GATS [42] & 2010 \\
\hline
\end{tabular}

DHS The Demographic and Health Surveys, ICS Individual Country Survey, GATS Global Adult Tobacco Survey, SEBS The Special Europe Barometer Survey, STEPS STEPwise approach to Surveillance

${ }^{\text {aP }}$ Populations of St Kitts and Nevis are tiny and unlikely to affect our estimates

(Additional file 1: Appendix 4). Based on the above reviews, we assumed that a causal association exists between some SLT products and cancers of the mouth, pharynx, and oesophagus, and ischaemic heart disease.

\section{Relative risks}

Based on 32 studies, the estimated pooled non-specific relative risk for mouth (oral cavity, tongue, and lip) cancers was 3.43 (95 \% CI 2.26-5.19) (Fig. 2). Studies from South-East Asia indicated an increased risk of oral cancer for SLT use whereas results from studies pertaining to Europe and the Americas did not substantiate such an association. For cancers of the pharynx, pooled nonspecific relative risk was 2.23 (95\% CI 1.55-3.20), based on ten studies (Fig. 3). For oesophageal cancers, no clear increased risk was present in studies in the USA, whereas a pooled estimate reported a relative risk of 2.17 (95 \% CI 1.70-2.78) (Fig. 4). For ischaemic heart disease, no good country-specific risk estimates were available (Fig. 5). However, we found one large casecontrol study (INTERHEART study) [29] conducted in 52 countries from all regions showing a statistically significant risk of ischaemic heart disease (adjusted odds ratio 1.57, 95 \% CI 1.24-1.99) among SLT users.

\section{Applying risk estimates}

For cancers in general, pooled country-specific risk estimates obtained from Sweden and the USA were applied 
Table 3 Smokeless tobacco use and risk of cancers, ischaemic heart disease, and stroke-studies included in meta-analysis

\begin{tabular}{|c|c|c|c|c|c|c|c|c|c|}
\hline Country & Study period & Study design & Exposure status & $\begin{array}{l}\text { Inclusion of } \\
\text { cigarette/alcohol } \\
\text { users }\end{array}$ & Outcome & $\begin{array}{l}\text { Odds ratios/relative risks } \\
\text { ( } 95 \% \text { confidence intervals) }\end{array}$ & Comments & $\begin{array}{l}\text { Quality assessment } \\
\text { (NOS) }^{\mathrm{a}}\end{array}$ & Reference \\
\hline \multicolumn{10}{|l|}{ CANCERS } \\
\hline \multirow[t]{3}{*}{ India } & \multirow[t]{3}{*}{$2001-2004$} & \multirow[t]{3}{*}{ Case-control } & \multirow{3}{*}{$\begin{array}{l}\text { Smokeless tobacco } \\
\text { with or without } \\
\text { additives }\end{array}$} & \multirow[t]{3}{*}{ No/No } & \multirow[t]{3}{*}{ Oral cancer } & \multirow[t]{3}{*}{$0.49(0.32-0.75)$} & \multirow[t]{3}{*}{ Exclusive SLT users } & Selection ${ }^{* * *}$ & \multirow{3}{*}{$\begin{array}{l}\text { Anantharaman } \\
\text { et al. } 2007 \text { [46] }\end{array}$} \\
\hline & & & & & & & & Comparability** & \\
\hline & & & & & & & & Exposure/Outcome* & \\
\hline \multirow[t]{3}{*}{ India } & \multirow[t]{3}{*}{ 1996-1999 } & \multirow[t]{3}{*}{ Case-control } & \multirow[t]{3}{*}{ Ever SLT users } & \multirow[t]{3}{*}{ Yes/Yes } & \multirow[t]{3}{*}{ Oral cancer } & $7.31(3.79-14.1)$ & $\begin{array}{l}\text { Never drinkers } \\
\text { adjusted for } \\
\text { smoking }\end{array}$ & Selection $* * * *$ & \multirow[t]{3}{*}{$\begin{array}{l}\text { Balaram et al. } \\
2002[47]\end{array}$} \\
\hline & & & & & & \multirow[t]{2}{*}{$9.19(4.38-19.28)$} & \multirow{2}{*}{$\begin{array}{l}\text { Never smokers } \\
\text { adjusted for } \\
\text { alcohol }\end{array}$} & Comparability** & \\
\hline & & & & & & & & Exposure/Outcome * & \\
\hline \multirow[t]{3}{*}{ India } & \multirow[t]{3}{*}{ 1982-1992 } & \multirow[t]{3}{*}{ Case-control } & \multirow{3}{*}{$\begin{array}{l}\text { Tobacco quid } \\
\text { chewing }\end{array}$} & \multirow[t]{3}{*}{ Yes/No } & Oral cancer & $5.8(3.6-9.34)$ & \multirow{3}{*}{$\begin{array}{l}\text { Adjusted for } \\
\text { smoking }\end{array}$} & Selection*** & \multirow{3}{*}{$\begin{array}{l}\text { Dikshit \& Kanhere } \\
2000 \text { [48] }\end{array}$} \\
\hline & & & & & Pharyngeal cancer & $1.2(0.8-1.8)$ & & Comparability* & \\
\hline & & & & & Lung cancer & $0.7(0.4-1.22)$ & & Exposure/Outcome* & \\
\hline \multirow[t]{3}{*}{ India } & \multirow[t]{3}{*}{ Unclear } & \multirow[t]{3}{*}{ Case-control } & Chewing tobacco & No/No & Oral cancer & $10.75(6.58-17.56)$ & Exclusive SLT users & Selection** & Goud et al. \\
\hline & & & & & & & & Comparability* & \\
\hline & & & & & & & & Exposure/Outcome ${ }^{0}$ & \\
\hline India & 1990-1997 & Cohort & Current SLT users & No/No & Oral cancer & $5.5(3.3-9.17)$ & Exclusive SLT users & Selection $* * * *$ & Jayalekshmi et al. \\
\hline & & & Former SLT users & & & $9.2(4.6-18.40)$ & & Comparability* & \\
\hline & & & & & & & & Exposure/Outcome** & \\
\hline India & 1990-1997 & Cohort & Current SLT user & Yes/Yes & Oral cancer & $2.4(1.7-3.39)$ & Adjusted for & Selection**** & Jayalekshmi et al. \\
\hline & & & Former SLT users & & & $2.1(1.3-3.39)$ & $\begin{array}{l}\text { smoking and } \\
\text { alcohol }\end{array}$ & Comparability* & 2010 [51] \\
\hline & & & & & & & & Exposure/Outcome*** & \\
\hline India & May 2005 & Case-control & Ever SLT users & No/No & Oral cancer & $4.23(3.11-5.75)$ & Exclusive SLT users & Selection*** & Jayant et al. \\
\hline & & & & & Pharyngeal cancer & $2.42(1.74-3.37)$ & & Comparability** & \\
\hline & & & & & Laryngeal cancer & $2.8(2.07-3.79)$ & & Exposure/Outcome $e^{0}$ & \\
\hline & & & & & Oesophageal cancer & $1.55(1.15-2.07)$ & & & \\
\hline India & 1968 & Case-control & Tobacco & Yes/No & Oral cancer & $4.63(3.50-6.14)$ & Exclusive chewers & Selection*** & Jussawalla \& \\
\hline & & & & & Pharyngeal cancer & $3.09(2.31-4.13)$ & $\begin{array}{l}\text { and non-chewers } \\
\text { data available }\end{array}$ & Comparability** & $\begin{array}{l}\text { Deshpande } 1971 \\
\text { [53] }\end{array}$ \\
\hline & & & & & Laryngeal cancer & $2.29(1.72-3.05)$ & & Exposure/Outcome ${ }^{0}$ & \\
\hline & & & & & Oesophageal cancer & $3.82(2.84-5.13)$ & & & \\
\hline India & $2005-2006$ & Case-control & Tobacco flakes & Yes/Yes & Oral cancer & $7.6(4.9-11.79)$ & Adjusted for & Selection**** & Madani et al. \\
\hline & & & Gutkha & & & $12.7(7-23.04)$ & $\begin{array}{l}\text { smoking and } \\
\text { alcohol }\end{array}$ & Comparability** & 2010 [54] \\
\hline & & & Mishiri & & & $3.0(1.9-4.74)$ & & Exposure/Outcome* & \\
\hline
\end{tabular}


Table 3 Smokeless tobacco use and risk of cancers, ischaemic heart disease, and stroke — studies included in meta-analysis (Continued)

\begin{tabular}{|c|c|c|c|c|c|c|c|c|c|}
\hline \multirow[t]{3}{*}{ India } & \multirow[t]{3}{*}{ Unclear } & \multirow[t]{3}{*}{ Case-control } & \multirow[t]{3}{*}{ Chewing tobacco } & \multirow[t]{3}{*}{ Yes/Yes } & \multirow[t]{3}{*}{ Oral cancer } & \multirow[t]{3}{*}{$5.0(3.6-6.94)$} & \multirow{3}{*}{$\begin{array}{l}\text { Adjusted for } \\
\text { smoking and } \\
\text { alcohol }\end{array}$} & Selection**** & \multirow{3}{*}{$\begin{array}{l}\text { Muwonge et al. } \\
2008 \text { [55] }\end{array}$} \\
\hline & & & & & & & & Comparability* & \\
\hline & & & & & & & & Exposure/Outcome* & \\
\hline \multirow[t]{3}{*}{ India } & \multirow[t]{3}{*}{ 1982-1984 } & \multirow[t]{3}{*}{ Case-control } & \multirow[t]{3}{*}{ Chewing tobacco } & \multirow[t]{3}{*}{ Yes/No } & \multirow[t]{3}{*}{ Oral cancer } & \multirow[t]{3}{*}{$10.2(2.6-40.02)$} & \multirow{3}{*}{$\begin{array}{l}\text { Adjusted for } \\
\text { smoking }\end{array}$} & Selection*** & \multirow{3}{*}{$\begin{array}{l}\text { Nandakumar } \\
\text { et al. } 1990 \text { [56] }\end{array}$} \\
\hline & & & & & & & & Comparability $^{* *}$ & \\
\hline & & & & & & & & Exposure/Outcome* & \\
\hline \multirow[t]{3}{*}{ India } & \multirow[t]{3}{*}{ 1980-1984 } & \multirow[t]{3}{*}{ Case-control } & \multirow[t]{3}{*}{ SLT users } & \multirow[t]{3}{*}{ No/No } & \multirow[t]{3}{*}{ Oral cancer } & \multirow[t]{3}{*}{$1.99(1.41-2.81)$} & \multirow[t]{3}{*}{ Exclusive SLT users } & Selection** & \multirow{3}{*}{$\begin{array}{l}\text { Rao et al. } \\
1994 \text { [57] }\end{array}$} \\
\hline & & & & & & & & Comparability $^{0}$ & \\
\hline & & & & & & & & Exposure/Outcome* & \\
\hline \multirow[t]{3}{*}{ India } & \multirow[t]{3}{*}{ 1952-1954 } & Case-control & Chewing tobacco & No/No & Oral cancer & $4.85(2.32-10.14)$ & Exclusive SLT users & Selection*** & Sanghvi et al. \\
\hline & & & & & Pharyngeal cancer & $2.02(0.94-4.33)$ & & Comparability** & 1955 [58] \\
\hline & & & & & Laryngeal cancer & $0.76(0.37-1.56)$ & & Exposure/Outcome $^{0}$ & \\
\hline India & 1983-1984 & Case-control & Snuff (males only) & Yes/Yes & Oral cancer & $2.93(0.98-8.76)$ & Adjusted for & Selection*** & Sankaranarayan \\
\hline & & & & & & & $\begin{array}{l}\text { Smoking and } \\
\text { alcohol; adjusted }\end{array}$ & Comparability $^{0}$ & \\
\hline & & & & & & & $\begin{array}{l}\text { effect size is only } \\
\text { among males }\end{array}$ & Exposure/Outcome* & \\
\hline India & Not given & Case-control & Tobacco chewing & Yes/Yes & Oropharyngeal cancer & $7.98(4.11-13.58)^{b}$ & Adjusted for & Selection*** & Wasnik et al. \\
\hline & & & & & & & $\begin{array}{l}\text { Smoking and } \\
\text { alcohol }\end{array}$ & Comparability** & 1998 [00] \\
\hline & & & & & & & & Exposure/Outcome ${ }^{0}$ & \\
\hline India & $1991-2003$ & Case-control & Chewing tobacco & No/No & Oral cancer & $5.88(3.66-7.93)$ & Exclusive SLT users & Selection**** & Subapriya e al. \\
\hline & & & & & & & & Comparability** & \\
\hline & & & & & & & & Exposure/Outcome** & \\
\hline India & 1950-1962 & Case-control & $\begin{array}{l}\text { Tobacco with or } \\
\text { without paan or }\end{array}$ & Yes/No & $\begin{array}{l}\text { Oral and oropharyngeal } \\
\text { cancer }\end{array}$ & $41.90(34.20-51.33)$ & $\begin{array}{l}\text { Exclusive chewer } \\
\text { data available }\end{array}$ & Selection** & $\begin{array}{l}\text { Wahi et al. } \\
1965 \text { [62] }\end{array}$ \\
\hline & & & lime & & & & Note: data of habit & Comparability** & \\
\hline & & & & & & & $\begin{array}{l}\text { was not available } \\
\text { for the whole } \\
\text { cohort }\end{array}$ & Exposure/Outcome ${ }^{0}$ & \\
\hline Pakistan & 1996-1998 & Case-control & Naswar & Yes/Yes & Oral cancer & $9.53(1.73-52.50)$ & Adjusted for & Selection*** & Merchant et al. \\
\hline & & & Paan with tobacco & & & $8.42(2.31-30.69)$ & $\begin{array}{l}\text { Smoking and } \\
\text { alcohol }\end{array}$ & Comparability** & 2000 [63] \\
\hline & & & & & & & & Exposure/Outcome* & \\
\hline Sweden & 1973-2002 & Cohort & Snus & Yes/Yes & Oral and pharyngeal & $3.10(1.50-6.41)$ & Adjusted for & Selection** & Roosar et al. \\
\hline & & & & & & & alcohol & Comparability** & $2000[04]$ \\
\hline & & & & & & & & Outcome $e^{* * *}$ & \\
\hline
\end{tabular}


Table 3 Smokeless tobacco use and risk of cancers, ischaemic heart disease, and stroke — studies included in meta-analysis (Continued)

\begin{tabular}{|c|c|c|c|c|c|c|c|c|c|}
\hline \multirow[t]{3}{*}{ India } & \multirow[t]{3}{*}{ 1993-1999 } & \multirow[t]{3}{*}{ Case-control } & \multirow[t]{3}{*}{ Chewing tobacco } & \multirow[t]{3}{*}{ Yes/Yes } & Oral cancer & $5.05(4.26-5.99)$ & \multirow{3}{*}{$\begin{array}{l}\text { Adjusted for } \\
\text { smoking and } \\
\text { alcohol }\end{array}$} & Selection ${ }^{* * *}$ & \multirow{3}{*}{$\begin{array}{l}\text { Znaor et al. } \\
2003 \text { [65] }\end{array}$} \\
\hline & & & & & Pharynx & $1.83(1.43-2.34)$ & & Comparability** & \\
\hline & & & & & Oesophagus & $2.06(1.62-2.62)$ & & Exposure/Outcome* & \\
\hline \multirow[t]{4}{*}{ Norway } & \multirow[t]{4}{*}{$1966-2001$} & \multirow[t]{4}{*}{ Cohort } & \multirow{4}{*}{$\begin{array}{l}\text { Chewing tobacco } \\
\text { plus oral snuff }\end{array}$} & \multirow[t]{4}{*}{ No/No } & Oral cancer & $1.1(0.5-2.42)$ & \multirow{4}{*}{$\begin{array}{l}\text { Adjusted for } \\
\text { smoking, might } \\
\text { be confounded } \\
\text { by alcohol use }\end{array}$} & Selection*** & \multirow{4}{*}{$\begin{array}{l}\text { Bofetta et al. } \\
2005 \text { [66] }\end{array}$} \\
\hline & & & & & Oesophageal cancer & $1.4(0.61-3.21)$ & & Comparability* & \\
\hline & & & & & Pancreatic cancer & $1.67(1.12-2.49)$ & & Exposure/Outcome ${ }^{* * *}$ & \\
\hline & & & & & Lung cancer & $0.80(0.61-1.05)$ & & & \\
\hline \multirow[t]{4}{*}{ Sweden } & \multirow[t]{4}{*}{ 1988-1991 } & \multirow[t]{4}{*}{ Case-control } & \multirow[t]{4}{*}{ Oral snuff } & \multirow[t]{4}{*}{ Yes/Yes } & Oral cancer & $1.4(0.8-2.45)$ & \multirow{4}{*}{$\begin{array}{l}\text { Adjusted for } \\
\text { smoking and } \\
\text { alcohol }\end{array}$} & Selection** & \multirow{4}{*}{$\begin{array}{l}\text { Lewin et al. } \\
1998 \text { [67] }\end{array}$} \\
\hline & & & & & Larynx & $0.9(0.5-1.62)$ & & Comparability** & \\
\hline & & & & & Oesophagus & $1.2(0.7-2.06)$ & & Exposure/Outcome* & \\
\hline & & & & & Pharynx & $0.7(0.4-1.22)$ & & & \\
\hline \multirow[t]{3}{*}{ Sweden } & \multirow[t]{3}{*}{ 1969-1992 } & \multirow[t]{3}{*}{ Cohort } & \multirow[t]{3}{*}{ Snus } & \multirow[t]{3}{*}{ No/No } & Oral cancer & $0.8(0.4-1.60)$ & \multirow[t]{3}{*}{ Exclusive SLT users } & Selection*** & \multirow{3}{*}{$\begin{array}{l}\text { Luo et al. } \\
2007 \text { [68] }\end{array}$} \\
\hline & & & & & Lung cancer & $0.8(0.5-1.28)$ & & Comparability* & \\
\hline & & & & & Pancreatic cancer & $2(1.20-3.33)$ & & Exposure/Outcome ${ }^{* * *}$ & \\
\hline Sweden & 2000-2004 & Case-control & Oral snuff & Yes/Yes & Oral & $0.70(0.3-1.63)$ & Adjusted for & Selection*** & Rosenquist et al \\
\hline & & & & & & & $\begin{array}{l}\text { Smoking and } \\
\text { alcohol }\end{array}$ & Comparability** & \\
\hline & & & & & & & & Exposure/Outcome ${ }^{* *}$ & \\
\hline Sweden & 1980-1989 & Case-control & Oral snuff & Yes/Yes & Oral cancer & $0.8(0.5-1.28)$ & Adjusted for & Selection** & Schildt et al. \\
\hline & & & & & & & $\begin{array}{l}\text { Smoking and } \\
\text { alcohol }\end{array}$ & Comparability** & 1998 [/0] \\
\hline & & & & & & & & Exposure/Outcome*** & \\
\hline USA & 1972-1983 & Case-control & Oral snuff & Yes/Yes & Oral cancer & $0.8(0.4-1.60)$ & Not clear if & Selection** & Mashberg et al. \\
\hline & & & Chewing tobacco & & & $1(0.7-1.43)$ & $\begin{array}{l}\text { adjusted for } \\
\text { smoking and }\end{array}$ & Comparability $^{0}$ & 1993 [71] \\
\hline & & & & & & & alcohol & Exposure/Outcome* & \\
\hline USA & Not given & Case-control & SLT use & Yes/Yes & Oral cancer & $0.90(0.38-2.13)$ & Adjusted for & Selection*** & Zhou et al. 2013 [15] \\
\hline & & & & & Pharyngeal cancer & $1.59(0.84-3.01)$ & $\begin{array}{l}\text { Smoking and } \\
\text { alcohol }\end{array}$ & Comparability** & \\
\hline & & & & & Laryngeal cancer & $0.67(0.19-2.36)$ & & Exposure/Outcome* & \\
\hline India & 2001-2004 & Case-control & Chewing tobacco & No/No & Pharyngeal cancer & $3.18(1.92-5.27)$ & Exclusive SLT users & Selection*** & Sapkota et al. \\
\hline & & & & & Laryngeal cancer & $0.95(0.52-1.74)$ & & Comparability** & \\
\hline & & & & & & & & Exposure/Outcome* & \\
\hline Pakistan & 1998-2002 & Case-control & Snuff dipping & No/No & Oesophageal cancer & $4.1(1.3-12.93)$ & Adjusted for & Selection*** & Akhtar et al. \\
\hline & & & Quid with tobacco & & & $14.2(6.4-31.50)$ & areca nut & Comparability** & 2012 [73] \\
\hline & & & & & & & & Exposure/Outcome ${ }^{* *}$ & \\
\hline
\end{tabular}


Table 3 Smokeless tobacco use and risk of cancers, ischaemic heart disease, and stroke-studies included in meta-analysis (Continued)

\begin{tabular}{|c|c|c|c|c|c|c|c|c|c|}
\hline \multirow[t]{3}{*}{ India } & \multirow[t]{3}{*}{ 2008-2012 } & \multirow[t]{3}{*}{ Case-control } & Nass chewing & \multirow[t]{3}{*}{ No/No } & \multirow[t]{3}{*}{ Oesophageal cancer } & $2.88(2.06-4.03)$ & \multirow[t]{3}{*}{ Exclusive SLT users } & Selection*** & \multirow{3}{*}{$\begin{array}{l}\text { Dar et al. } \\
2012[74]\end{array}$} \\
\hline & & & \multirow[t]{2}{*}{ Gutkha chewing } & & & \multirow[t]{2}{*}{$2.87(0.87-9.47)$} & & Comparability** & \\
\hline & & & & & & & & Exposure/Outcome & \\
\hline \multirow[t]{3}{*}{ India } & \multirow[t]{3}{*}{$2007-2011$} & \multirow[t]{3}{*}{ Case-control } & \multirow[t]{3}{*}{ Oral snuff } & \multirow[t]{3}{*}{ Yes/Yes } & \multirow[t]{3}{*}{ Oesophageal cancer } & \multirow[t]{3}{*}{$3.86(2.46-6.06)$} & \multirow{3}{*}{$\begin{array}{l}\text { Adjusted for } \\
\text { smoking and } \\
\text { alcohol }\end{array}$} & Selection** & \multirow{3}{*}{$\begin{array}{l}\text { Sehgal et al. } \\
2012[75]\end{array}$} \\
\hline & & & & & & & & Comparability $^{* *}$ & \\
\hline & & & & & & & & Exposure/Outcome* & \\
\hline \multirow[t]{3}{*}{ India } & \multirow[t]{3}{*}{ 2011-2012 } & \multirow[t]{3}{*}{ Case-control } & \multirow[t]{3}{*}{ Chewing tobacco } & \multirow[t]{3}{*}{ Yes/Yes } & \multirow[t]{3}{*}{ Oesophageal cancer } & \multirow[t]{3}{*}{$2.63(1.53-4.52)$} & \multirow{3}{*}{$\begin{array}{l}\text { Adjusted for } \\
\text { smoking and } \\
\text { alcohol }\end{array}$} & Selection*** & \multirow{3}{*}{$\begin{array}{l}\text { Talukdar et al. } \\
2013 \text { [76] }\end{array}$} \\
\hline & & & & & & & & Comparability** & \\
\hline & & & & & & & & Exposure/Outcome* & \\
\hline \multirow[t]{3}{*}{ Sweden } & \multirow[t]{3}{*}{ 1995-1997 } & Case-control & Oral snuff & Yes/Yes & $\begin{array}{l}\text { Oesophageal cancer } \\
\text { (adenocarcinoma) }\end{array}$ & $1.2(0.7-2.06)$ & $\begin{array}{l}\text { Adjusted for } \\
\text { smoking and }\end{array}$ & Selection*** & $\begin{array}{l}\text { Lagergren et al. } \\
2000 \text { [77] }\end{array}$ \\
\hline & & & & & (Squamous cell & $1.4(0.9-2.18)$ & & Comparability** & \\
\hline & & & & & carcinoma) & & & Exposure/Outcome* & \\
\hline Sweden & 1969-1993 & Cohort & Oral snuff & Yes/No & $\begin{array}{l}\text { Oesophageal cancer } \\
\text { (Adenocarcinoma) }\end{array}$ & $1.3(0.8-2.11)$ & $\begin{array}{l}\text { Adjusted for } \\
\text { smoking }\end{array}$ & Selection** & $\begin{array}{l}\text { Zendehdel et al. } \\
2008 \text { [78] }\end{array}$ \\
\hline & & & & & (Squamous cell & $1.2(0.8-1.80)$ & & Comparability* & \\
\hline & & & & & carcinoma) & & & Exposure/Outcome ${ }^{* *}$ & \\
\hline Sweden & 1974-1985 & Cohort & SLT users & No/NA & Lung cancer & $0.90(0.20-4.05)$ & Adjusted for age, & Selection*** & Bolinder et al. \\
\hline & & & & & & & region of origin & Comparability* & 1994 [79] \\
\hline & & & & & & & & Outcome $e^{* *}$ & \\
\hline Morocco & 1996-1998 & Case-control & SLT users & Yes/No & Lung cancer & $1.05(0.28-3.94)$ & Adjusted for & Selection** & Sasco et al. \\
\hline & & & & & & & smoking & Comparability** & $2002[80]$ \\
\hline & & & & & & & & Exposure/Outcome** & \\
\hline USA & 1977-1984 & Case-control & SLT users & Yes/No & Oesophageal cancer & $1.2(0.1-14.40)$ & Adjusted for & Selection ${ }^{* * *}$ & Brown et al. \\
\hline & & & & & & & & Comparability** & $1980[81]$ \\
\hline & & & & & & & & Exposure/Outcome ${ }^{* *}$ & \\
\hline USA & 1986-1989 & Case-control & SLT users & Yes/No & Pancreatic cancer & $1.4(0.5-3.92)$ & Adjusted for & Selection*** & Alguacil \& \\
\hline & & & & & & & & Comparability* & [82] \\
\hline & & & & & & & & Exposure/Outcome ${ }^{* *}$ & \\
\hline USA & $2000-2006$ & Case-control & Chewing tobacco & Yes/Yes & Pancreatic cancer & $0.6(0.3-1.20)$ & Adjusted for & Selection**** & Hassan et al. \\
\hline & & & Oral snuff & & & $0.5(0.1-2.5)$ & $\begin{array}{l}\text { smoking and } \\
\text { alcohol }\end{array}$ & Comparability** & $2007[83]$ \\
\hline & & & & & & & & Exposure/Outcome* & \\
\hline
\end{tabular}


Table 3 Smokeless tobacco use and risk of cancers, ischaemic heart disease, and stroke — studies included in meta-analysis (Continued)

\begin{tabular}{|c|c|c|c|c|c|c|c|c|c|}
\hline \multicolumn{10}{|c|}{ CARDIOVASCULAR DISEASES (ischaemic heart disease and stroke) } \\
\hline \multirow[t]{3}{*}{52 countries } & \multirow[t]{3}{*}{ 1999-2003 } & \multirow[t]{3}{*}{ Case-control } & \multirow[t]{3}{*}{ Chewing tobacco } & \multirow[t]{3}{*}{ No/Yes } & \multirow[t]{3}{*}{ Myocardial infarction } & \multirow[t]{3}{*}{$1.57(1.24-1.99)$} & \multirow{3}{*}{$\begin{array}{l}\text { Adjusted for } \\
\text { diabetes, abdominal } \\
\text { obesity, hypertension, } \\
\text { exercise, diet }\end{array}$} & Selection**** & \multirow{3}{*}{$\begin{array}{l}\text { Teo et al. } \\
2006 \text { [29] }\end{array}$} \\
\hline & & & & & & & & Comparability** & \\
\hline & & & & & & & & Exposure/Outcome* & \\
\hline \multirow[t]{3}{*}{ Pakistan } & \multirow[t]{3}{*}{$2005-2011$} & \multirow[t]{3}{*}{ Case-control } & Dippers only (Naswar) & \multirow[t]{3}{*}{ No/NA } & \multirow[t]{3}{*}{ Myocardial infarction } & $1.46(1.20-1.77)$ & \multirow{3}{*}{$\begin{array}{l}\text { Adjusted for age, sex, } \\
\text { region, ethnicity }\end{array}$} & Selection**** & \multirow{3}{*}{$\begin{array}{l}\text { Alexander } 2013 \\
\text { [84] }\end{array}$} \\
\hline & & & \multirow{2}{*}{$\begin{array}{l}\text { Chewers only } \\
\text { (Paan/ Supari/ Gutkha) }\end{array}$} & & & \multirow[t]{2}{*}{$1.71(1.46-2.00)$} & & Comparability** & \\
\hline & & & & & & & & Exposure/Outcome** & \\
\hline \multirow[t]{3}{*}{ Bangladesh } & \multirow[t]{3}{*}{$2006-2007$} & \multirow[t]{3}{*}{ Case-control } & \multirow[t]{3}{*}{ Ever SLT users } & \multirow[t]{3}{*}{ No/NA } & \multirow{3}{*}{$\begin{array}{l}\text { Myocardial infarction, } \\
\text { Angina pectoris }\end{array}$} & \multirow[t]{3}{*}{$2.8(1.1-7.13)$} & \multirow{3}{*}{$\begin{array}{l}\text { Adjusted for age, } \\
\text { sex, hypertension }\end{array}$} & Selection*** & Rahman \& Zaman \\
\hline & & & & & & & & Comparability** & \\
\hline & & & & & & & & Exposure/Outcome* & \\
\hline Bangladesh & 2010 & Case-control & Ever SLT users & No/NA & Myocardial infarction, & $0.77(0.52-1.14)$ & Adjusted for age, & Selection**** & Rahman et al. \\
\hline & & & & & Angina pectoris & & $\begin{array}{l}\text { hypertension, } \\
\text { diabetes, acute }\end{array}$ & Comparability ${ }^{* *}$ & 2012 [86] \\
\hline & & & & & & & psycho-social stress & Exposure/Outcome* & \\
\hline Sweden & $1998-2005$ & Case-control & Current SLT users & No/NA & Myocardial infarction & $0.73(0.35-1.52)$ & Exclusive SLT users & Selection*** & Hergens et al. \\
\hline & & & Former SLT users & & & $1.2(0.46-3.13)$ & & Comparability** & \\
\hline & & & & & & & & Exposure/Outcome** & \\
\hline Sweden & 1978-2004 & Cohort & Ever SLT users & No/NA & Myocardial infarction & $0.99(0.90-1.10)$ & Adjusted for age, & Selection** & Hergens et al. \\
\hline & & & & & & & $\begin{array}{l}\text { BMI, region of } \\
\text { residence }\end{array}$ & Comparability** & \\
\hline & & & & & & & & Exposure/Outcome $e^{* * *}$ & \\
\hline Sweden & 1989-1991 & Case-control & Regular SLT users & Yes/NA & Myocardial infarction & $1.01(0.66-1.55)^{c}$ & Adjusted for age, & Selection*** & Huhtasaari et al. \\
\hline & & & & & & & education, smoking & Comparability** & 1992 [89] \\
\hline & & & & & & & & Exposure/Outcome* & \\
\hline Sweden & 1991-1993 & Case-control & Former SLT users & No/NA & Myocardial infarction & $1.23(0.54-2.82)$ & Exclusive SLT users & Selection*** & Huhtasaari et al. \\
\hline & & & & & & & & Comparability** & \\
\hline & & & & & & & & Exposure/Outcome ${ }^{* *}$ & \\
\hline Sweden & 1988-2000 & Cohort & Daily SLT users & No/NA & Ischaemic heart disease & $1.41(0.61-3.28)$ & Adjusted for BMI, & Selection ${ }^{* * * *}$ & Johansson et al. \\
\hline & & & & & & & $\begin{array}{l}\text { diabetes, } \\
\text { divili }\end{array}$ & Comparability** & \\
\hline & & & & & & & hypertension & Exposure/Outcome** & \\
\hline Sweden & 1985-1999 & Case-control & Current SLT users & No/NA & Myocardial infarction & $0.82(0.46-1.46)$ & Adjusted for BMI, & Selection**** & Wennberg et al. \\
\hline & & & Former SLT users & & & $0.66(0.32-1.36)$ & $\begin{array}{l}\text { pnysical activity, } \\
\text { education, }\end{array}$ & Comparability** & \\
\hline & & & & & & & cholesterol & Exposure/Outcome** & \\
\hline Sweden & 1985-2000 & Case-control & Regular SLT users & No/NA & Stroke & $0.87(0.41-1.83)$ & Adjusted for diabetes, & Selection**** & Asplund et al. \\
\hline & & & & & & & $\begin{array}{l}\text { nypertension, } \\
\text { education, marital }\end{array}$ & Comparability** & 2003 [93] \\
\hline & & & & & & & status, cholesterol & Exposure/Outcome** & \\
\hline
\end{tabular}


Table 3 Smokeless tobacco use and risk of cancers, ischaemic heart disease, and stroke —studies included in meta-analysis (Continued)

\begin{tabular}{|c|c|c|c|c|c|c|c|c|c|}
\hline \multirow[t]{3}{*}{ Sweden } & \multirow[t]{3}{*}{ 1978-2003 } & \multirow[t]{3}{*}{ Cohort } & \multirow[t]{3}{*}{ Ever SLT users } & \multirow[t]{3}{*}{ No/NA } & \multirow[t]{3}{*}{ Stroke } & \multirow[t]{3}{*}{$1.02(0.92-1.13)$} & \multirow{3}{*}{$\begin{array}{l}\text { Adjusted for age, } \\
\text { BMl, region of } \\
\text { residence }\end{array}$} & Selection** & \multirow{3}{*}{$\begin{array}{l}\text { Hergens et al. } \\
2008 \text { [94] }\end{array}$} \\
\hline & & & & & & & & Comparability** & \\
\hline & & & & & & & & Exposure/Outcome**** & \\
\hline \multirow[t]{4}{*}{ Sweden } & \multirow[t]{4}{*}{ 1998-2005 } & \multirow[t]{4}{*}{ Cohort } & Current SLT users & \multirow[t]{4}{*}{ No/NA } & Ischaemic heart disease & $0.85(0.51-1.42)$ & \multirow{4}{*}{$\begin{array}{l}\text { Adjusted for age, } \\
\text { hypertension, } \\
\text { diabetes, cholesterol }\end{array}$} & Selection ${ }^{* * *}$ & \multirow{4}{*}{$\begin{array}{l}\text { Hansson et al. } \\
2009 \text { [95] }\end{array}$} \\
\hline & & & \multirow[t]{3}{*}{ Former SLT users } & & \multirow[t]{3}{*}{ Stroke } & $1.07(0.56-2.04)$ & & Comparability** & \\
\hline & & & & & & $1.18(0.67-2.08)$ & & Exposure/Outcome ${ }^{* *}$ & \\
\hline & & & & & & $1.35(0.65-2.82)$ & & & \\
\hline \multirow[t]{3}{*}{ Sweden } & \multirow[t]{3}{*}{ 1991-2004 } & \multirow[t]{3}{*}{ Cohort } & \multirow[t]{3}{*}{ SLT users } & \multirow[t]{3}{*}{ No/NA } & Myocardial infarction & $0.75(0.3-1.87)$ & \multirow{3}{*}{$\begin{array}{l}\text { Adjusted for age, } \\
\text { diabetes, occupation, } \\
\text { hypertension, physical } \\
\text { activity, BMI, marital } \\
\text { status }\end{array}$} & Selection*** & \multirow{3}{*}{$\begin{array}{l}\text { Janzon et al. } \\
2009 \text { [96] }\end{array}$} \\
\hline & & & & & \multirow[t]{2}{*}{ Stroke } & \multirow[t]{2}{*}{$0.59(0.2-1.5)$} & & Comparability** & \\
\hline & & & & & & & & Exposure/Outcome ${ }^{* *}$ & \\
\hline
\end{tabular}

BMI body mass index, NA not applicable, NOS Newcastle-Ottawa Scale, SLT smokeless tobacco

${ }^{a} \mathrm{NOS}$ for assessing the quality of non-randomised studies in meta-analyses based on selection, comparability, and exposure/outcome. Number of stars (*) indicates the number of criteria met for each of these three categories [23]

${ }^{b}$ Effect sizes are for oral and pharyngeal cancers combined and were included in the meta-analysis for oral cancer only

${ }^{\mathrm{C}}$ Based on parameter estimate and standard error reported in paper 


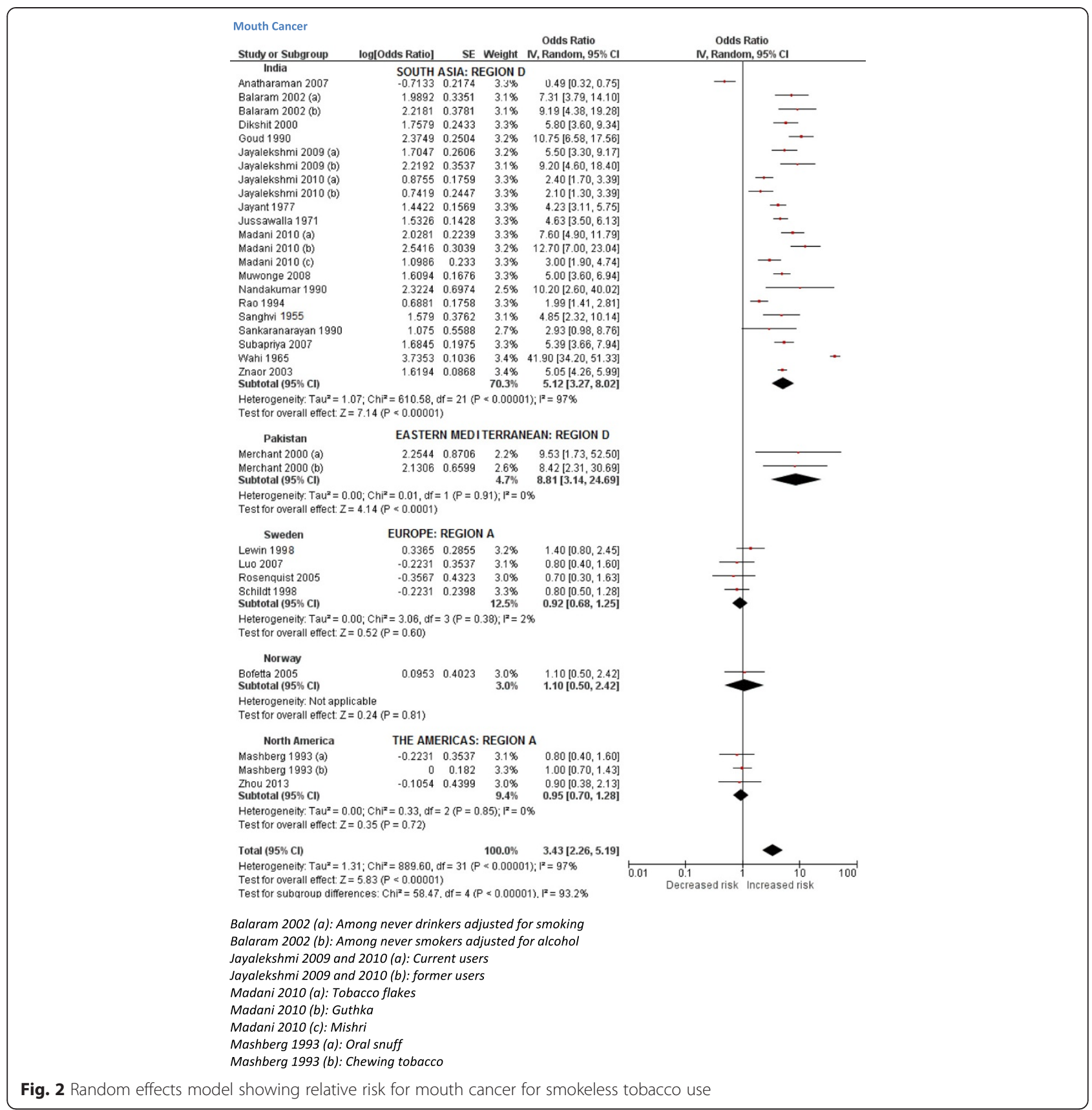

to Europe A and Americas A, respectively. For SouthEast Asia B and D and Western Pacific B regions, country-specific estimates from India were applied. There were a few exceptions to this rule, because some countries (UK, Mexico, Pakistan, China, Mongolia) differed in their SLT consumption patterns from their respective regions (see Additional file 1: Appendix 4 for details). In short, country-specific risk estimates for cancers could only be fully applied to five regions. For the remaining nine regions, our findings were imputed either by applying statistically significant non-specific risk estimates or none at all (Additional file 1: Appendix 4). In case of ischaemic heart disease, Sweden was the only country with a pooled country-specific relative risk $(0.98,95 \%$ CI 0.90-1.07) obtained from a good number (more than three) of studies. For 11 out of 14 regions, we used a large multi-country study (INTERHEART) - conducted in 52 countries-to apply and deduce risk estimates. The three regions (Europe A and C and Americas D) were excluded, as these were not among those regions included in the INTERHEART study (Additional file 1: Appendix 4). There was one exception (UK) where INTERHEART study estimates were applied because SLT products consumed in the UK commonly originate from South Asia. 


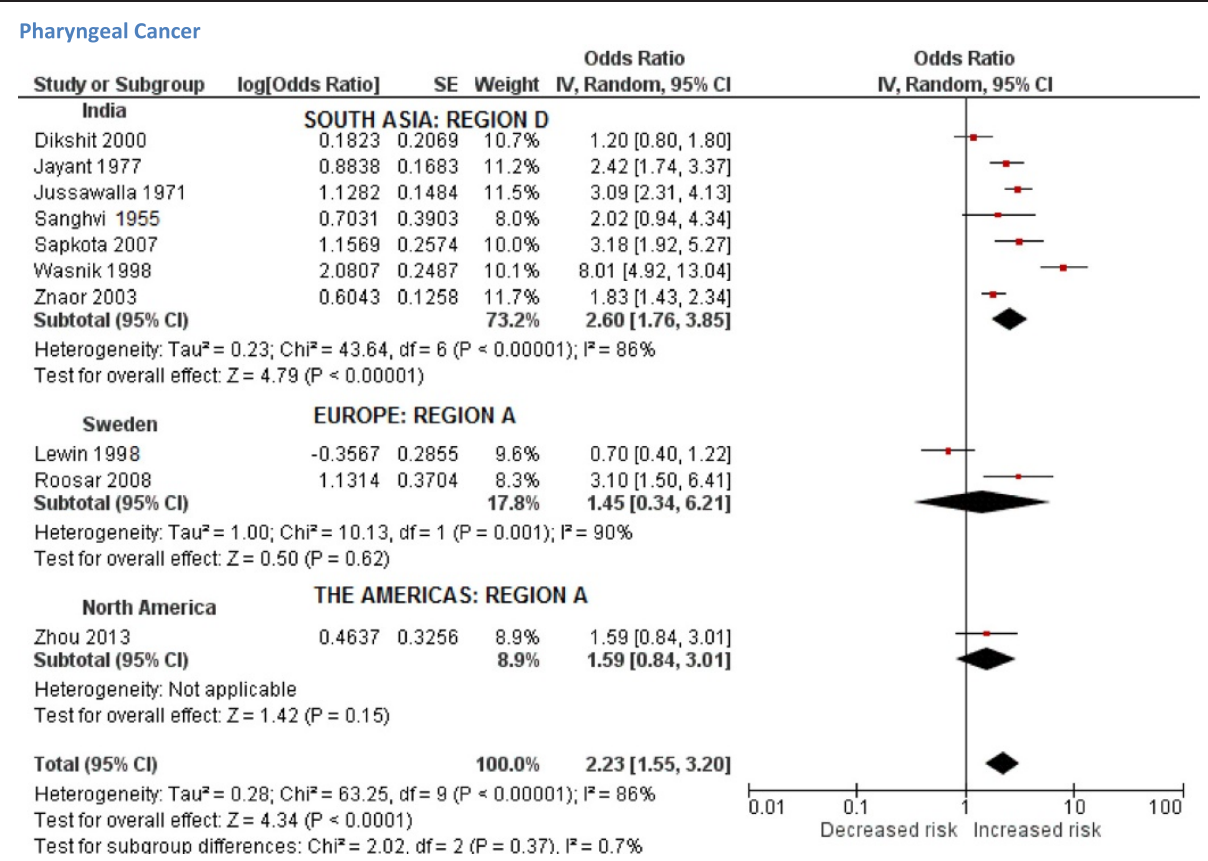

Fig. 3 Random effects model showing relative risk for pharyngeal cancer for smokeless tobacco use

\section{Attributable burden}

The attributable burden of SLT use is outlined in Table 4. Our estimates indicate that in 2010, SLT use led to $1,711,539$ DALYs lost and 62,283 deaths due to cancers of mouth, pharynx, and oesophagus, and, based on data from the benchmark 52 country INTERHEART study, 4,725,381 DALYs lost and 204,309 deaths from ischaemic heart disease. In total, SLT use caused the loss of $6,436,920$ DALYs and 266,592 deaths. The figures show that three-quarters of these deaths and loss of DALYs were among males. This disease burden was found to be distributed across all WHO sub-regions. However, nearly $85 \%$ of the total burden attributable to SLT use was in South-East Asia, with India alone accounting for $74 \%$ of the global burden, followed by Bangladesh (5\%).

\section{Discussion}

We have found that SLT is consumed worldwide and that its use results in substantial, potentially avoidable, morbidity and mortality. However, owing to marked differences in the types of products available, patterns of consumption, and associated risks, there are substantial differences in the attributable burden between regions and countries. In particular, SLT consumption in SouthEast Asia leads to a much greater burden of disease than in Sweden, despite its use being equally prevalent. This is due to the much lower levels of TSNA and pH in SLT products in Sweden compared to those found in SLT in South-East Asia [6]. Similarly, SLT products used in the
USA have lower risk estimates than for those used in South-East Asia.

We found that more than six million DALYs were lost and over a quarter of a million deaths occurred in 2010 owing to SLT consumption. However, our estimates require cautious interpretation because of a number of potential limitations.

First, our analysis was limited to those countries and diseases for which reliable prevalence and risk data were available, respectively. Most global tobacco surveys that reported on SLT consumption did not include all countries in the world. While global figures on smoking prevalence were available, we did not find any SLT prevalence figures for almost half of all countries. Where SLT prevalence figures were available, two countries (Micronesia and Saint Kitts \& Nevis) were excluded from the final estimates owing to an absence of data for cancers in the 2010 Global Burden of Disease study. Moreover, for certain disease outcomes, e.g. adverse reproductive and oral health effects, poor quality as well as limited quantity of evidence precluded their inclusion.

Second, lack of country-specific risk estimates leads to considerable uncertainty. Despite several countries reporting SLT consumption, most did not have any reliable information on the types of SLT products used and on their associated health risks. For example, studies from several African countries reported high SLT consumption (Table 2), but provided little information on their hazard profile. There is some evidence, mainly 


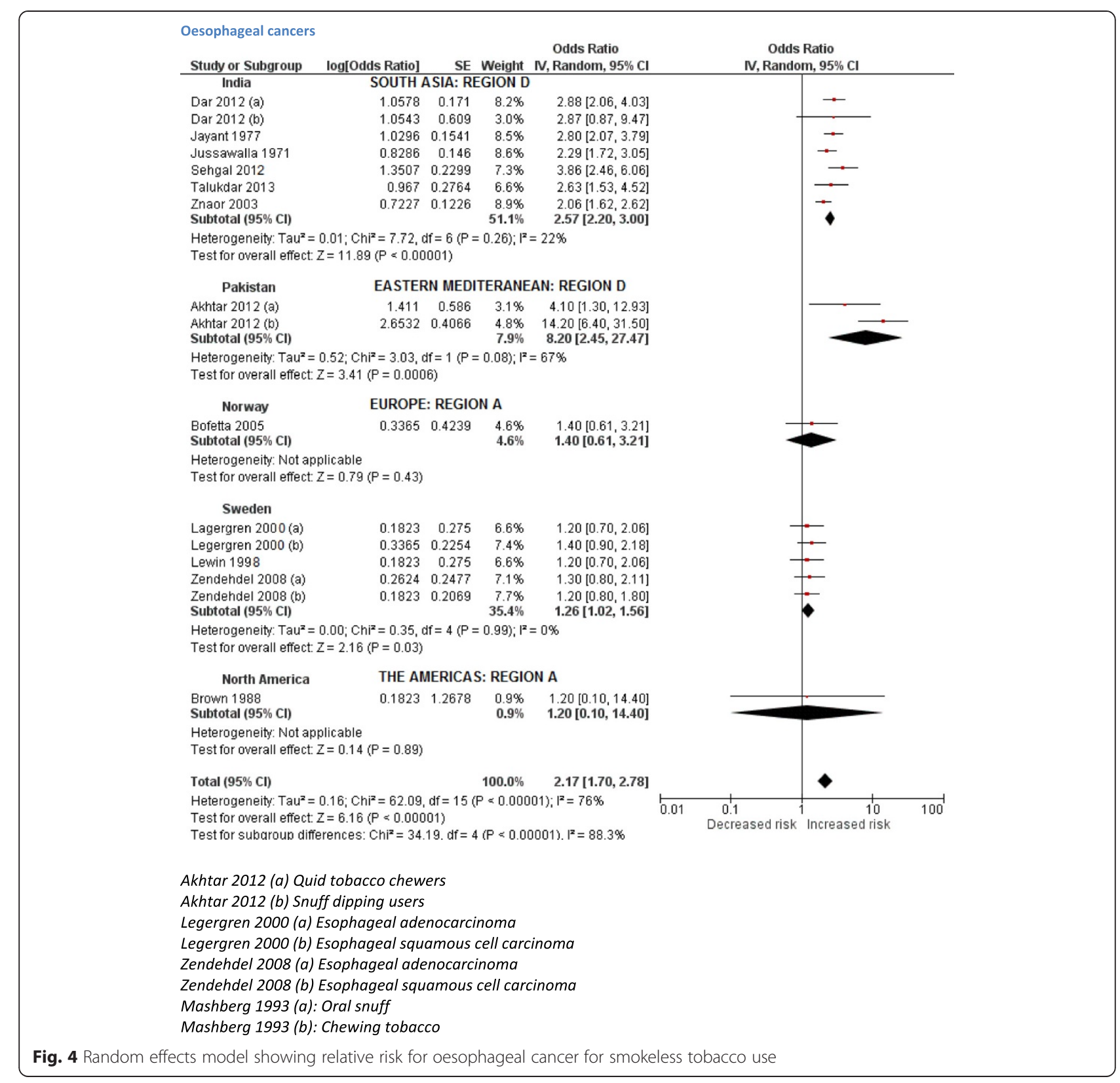

from Sudan [30], that products used in Africa tend to have a higher $\mathrm{pH}$ than those used in Europe or in the USA. However, we did not find any data on the risks associated with widespread SLT use in southern parts of Africa. Likewise, various forms of SLT have been used in parts of South America (Brazilian rapê or Venezuelan chimó) for many years, yet there are no studies on the health effects of such products. In the absence of country-specific risk estimates, we assumed that in general those populations that consume similar SLT products are likely to share similar health risks and susceptibilities. We extrapolated and applied risk estimates to most countries included in our analysis on that basis (Additional file 1: Appendix 4). For cancer, our extrapolation was based on estimates obtained from several studies; for ischaemic heart diseases, extrapolations were mostly based on a single although large multicountry study (INTERHEART). As a result, almost three-quarters of the estimated SLT disease burden, which is attributed to ischaemic heart disease, is uncertain. Therefore, a cautious interpretation would be to exclude ischaemic heart disease burden figures from our estimates. However, in estimating these figures we had already excluded those regions and their respective countries that were not included in INTERHEART study. As a pointer on future research, our study 


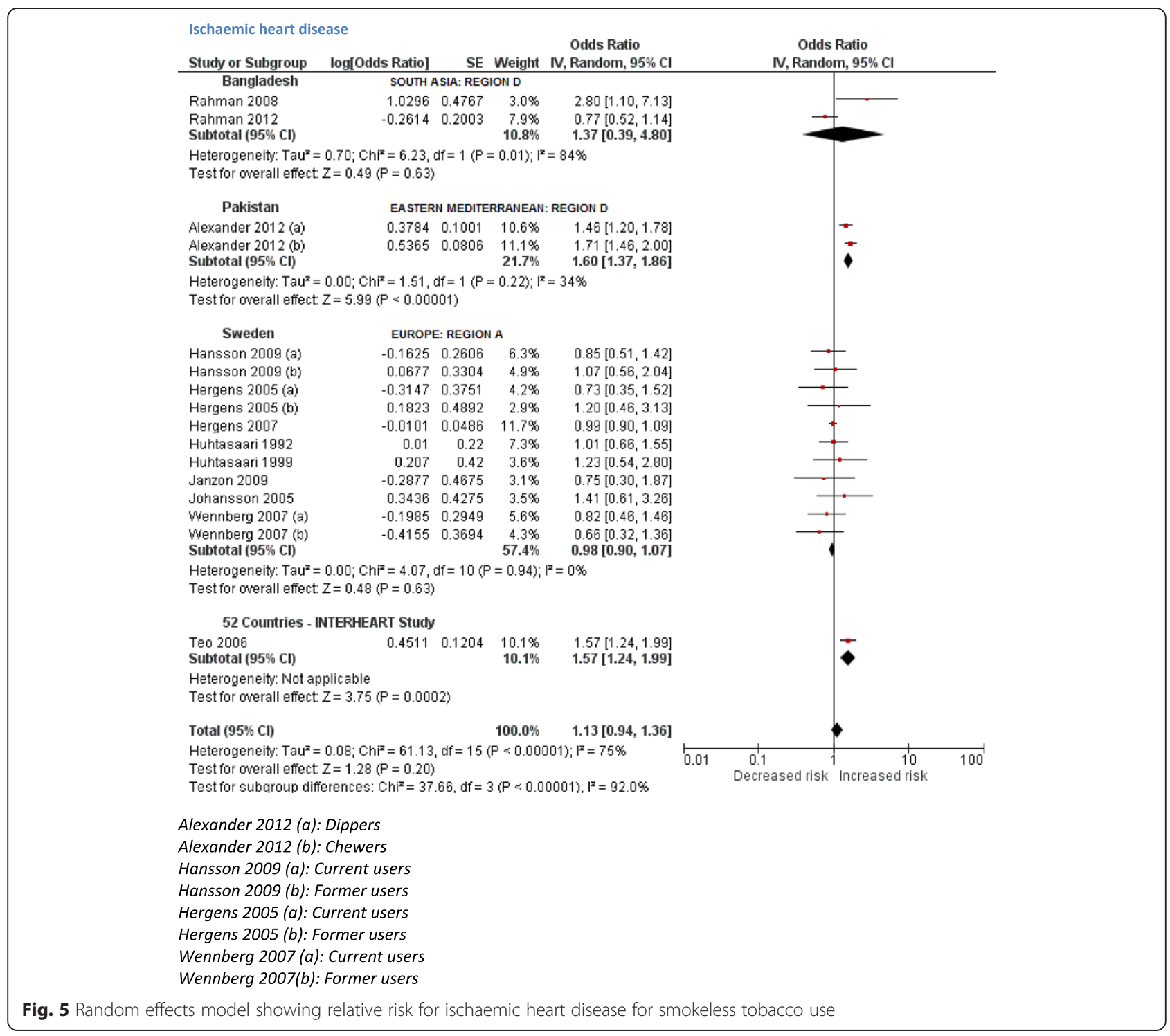

highlights the need to study risk of SLT consumption on ischaemic heart diseases across the spectrum of SLT products and consumption behaviours. In time, this will produce more country-specific risk estimates, which would undoubtedly improve the reliability of our estimates presented here.

Third, the disease burden observed in 2010 is unlikely to be a consequence of SLT consumption in recent years. Therefore, our prevalence figures, obtained in surveys carried out in the last decade and used in the estimates, could be problematic. However, we assumed that the SLT consumption rates have remained stable over the last 30-40 years in these countries. We consider this as a safe assumption given that SLT use is not a new trend and historically embedded in culture and tradition in many countries, most remarkably in South Asia [31]. Consumption trends based on repeated youth surveys in
India and Bangladesh suggest that SLT use has remained stable over the last decade [32]. Evidence from Sweden suggests that while more people are using snus now than 25 years ago, the consumption trends, compared to cigarette use, have essentially remained stable in this period [33, 34].

Finally, the age range of the adult sampling frames used in different SLT prevalence surveys varied, which could also increase uncertainty. The main difference between two of the key categories used was in the adult range starting from either $\geq 15$ years or $\geq 25$ years. Given that the risk of cancers and ischemic heart disease accumulates after many years of use well beyond young adult age, it may not have made much of a difference to our burden of disease estimates.

For the seven countries in South-East Asia region D, we estimated that 55,060 deaths caused by cancers of 
Table 4 Number of DALYs lost and deaths from SLT use in 2010, by WHO sub-region as defined in Additional file 1: Appendix 1

\begin{tabular}{|c|c|c|c|c|c|c|c|c|c|c|c|c|c|c|c|}
\hline \multirow[t]{2}{*}{ WHO sub-regions ${ }^{a}$} & \multicolumn{3}{|c|}{ Mouth cancer } & \multicolumn{3}{|c|}{ Pharyngeal cancer } & \multicolumn{3}{|c|}{ Oesophageal cancer } & \multicolumn{3}{|c|}{ Ischaemic heart disease } & \multicolumn{3}{|l|}{ All causes } \\
\hline & $\mathrm{M}$ & $\mathrm{F}$ & All & M & $\mathrm{F}$ & All & M & $F$ & All & $\mathrm{M}$ & $\mathrm{F}$ & All & $\mathrm{M}$ & $\mathrm{F}$ & All \\
\hline \multicolumn{16}{|l|}{ DEATHS } \\
\hline Africa D & 86 & 36 & 123 & 15 & 2 & 17 & 157 & 77 & 233 & 2323 & 751 & 3074 & 2581 & 866 & 3448 \\
\hline Africa $E$ & 155 & 85 & 240 & 19 & 12 & 31 & 389 & 252 & 641 & 1202 & 923 & 2125 & 1765 & 1272 & 3037 \\
\hline Americas A & 0 & 0 & 0 & 0 & 0 & 0 & 0 & 0 & 0 & 10,240 & 649 & 10,889 & 10,240 & 649 & 10,889 \\
\hline Americas B & 90 & 11 & 102 & 28 & 3 & 31 & 74 & 9 & 83 & 1030 & 291 & 1321 & 1222 & 314 & 1536 \\
\hline Americas D & 0 & 0 & 0 & 0 & 0 & 0 & 0 & 0 & 0 & 0 & 0 & 0 & 0 & 0 & 0 \\
\hline Eastern Mediterranean B & 11 & 1 & 12 & 1 & 0 & 2 & 4 & 1 & 5 & 441 & 74 & 515 & 457 & 76 & 534 \\
\hline Eastern Mediterranean D & 933 & 254 & 1187 & 604 & 59 & 663 & 1012 & 129 & 1141 & 7401 & 926 & 8327 & 9950 & 1368 & 11,318 \\
\hline Europe A & 66 & 13 & 78 & 16 & 2 & 18 & 244 & 38 & 282 & 539 & 145 & 684 & 865 & 197 & 1062 \\
\hline Europe B & 146 & 3 & 148 & 57 & 1 & 58 & 260 & 2 & 262 & 5506 & 156 & 5662 & 5969 & 162 & 6130 \\
\hline Europe C & 0 & 0 & 0 & 0 & 0 & 0 & 0 & 0 & 0 & 0 & 0 & 0 & 0 & 0 & 0 \\
\hline South-East Asia B & 438 & 396 & 835 & 129 & 58 & 187 & 243 & 139 & 382 & 3205 & 1852 & 5057 & 4016 & 2445 & 6461 \\
\hline South-East Asia D & 11,527 & 6459 & 17,987 & 12,715 & 3485 & 16,200 & 15,247 & 5625 & 20,873 & 117,523 & 45,047 & 162,570 & 157,013 & 60,617 & 217,630 \\
\hline Western Pacific A & 0 & 0 & 0 & 0 & 0 & 0 & 0 & 0 & 0 & 69 & 36 & 104 & 69 & 36 & 104 \\
\hline Western Pacific B & 134 & 159 & 293 & 22 & 34 & 56 & 51 & 63 & 114 & 3167 & 814 & 3981 & 3374 & 1070 & 4443 \\
\hline Worldwide & 13,586 & 7418 & 21,003 & 13,608 & 3656 & 17,264 & 17,680 & 6336 & 24,016 & 152,647 & 51,662 & 204,309 & 197,520 & 69,072 & 266,592 \\
\hline \multicolumn{16}{|l|}{ DALYS } \\
\hline Africa D & 2516 & 1046 & 3562 & 452 & 65 & 517 & 4119 & 1906 & 6024 & 64,043 & 19,116 & 83,159 & 71,130 & 22,132 & 93,262 \\
\hline Africa E & 4926 & 2293 & 7220 & 573 & 349 & 922 & 10,159 & 6290 & 16,449 & 33,502 & 21,109 & 54,610 & 49,159 & 30,042 & 79,201 \\
\hline Americas A & 0 & 0 & 0 & 0 & 0 & 0 & 0 & 0 & 0 & 172,206 & 7213 & 179,419 & 172,206 & 7213 & 179,419 \\
\hline Americas B & 2311 & 230 & 2541 & 734 & 63 & 797 & 1717 & 176 & 1893 & 22,252 & 4728 & 26,980 & 27,014 & 5197 & 32,210 \\
\hline Americas D & 0 & 0 & 0 & 0 & 0 & 0 & 0 & 0 & 0 & 0 & 0 & 0 & 0 & 0 & 0 \\
\hline Eastern Mediterranean B & 285 & 36 & 321 & 33 & 9 & 43 & 86 & 20 & 106 & 9841 & 1383 & 11,224 & 10,246 & 1448 & 11,694 \\
\hline Eastern Mediterranean D & 29,240 & 7669 & 36,909 & 16,446 & 1800 & 18,247 & 27,777 & 3613 & 31,390 & 187,394 & 21,544 & 208,938 & 260,857 & 34,627 & 295,483 \\
\hline Europe A & 1514 & 224 & 1738 & 369 & 45 & 414 & 4949 & 545 & 5494 & 8397 & 1491 & 9888 & 15,230 & 2304 & 17,534 \\
\hline Europe B & 4439 & 60 & 4499 & 1704 & 20 & 1724 & 6460 & 56 & 6517 & 115,640 & 1991 & 117,631 & 128,243 & 2128 & 130,371 \\
\hline Europe C & 0 & 0 & 0 & 0 & 0 & 0 & 0 & 0 & 0 & 0 & 0 & 0 & 0 & 0 & 0 \\
\hline South-East Asia B & 10,968 & 7741 & 18,709 & 3217 & 1487 & 4704 & 5608 & 2983 & 8591 & 66,969 & 29,913 & 96,881 & 86,762 & 42,124 & 128,886 \\
\hline South-East Asia D & 351,752 & 179,051 & 530,803 & 338,976 & 107,041 & 446,017 & 400,770 & 143,146 & 543,916 & 290,6993 & 938,528 & $3,845,521$ & $3,998,491$ & $1,367,766$ & $5,366,257$ \\
\hline Western Pacific A & 0 & 0 & 0 & 0 & 0 & 0 & 0 & 0 & 0 & 1024 & 340 & 1364 & 1024 & 340 & 1364 \\
\hline Western Pacific B & 3700 & 3567 & 7267 & 615 & 794 & 1409 & 1313 & 1485 & 2797 & 72,936 & 16,830 & 89,766 & 78,564 & 22,675 & 101,239 \\
\hline Worldwide & 411,652 & 201,918 & 613,569 & 363,120 & 111,673 & 474,793 & 462,957 & 160,219 & 623,177 & $3,661,195$ & $1,064,186$ & $4,725,381$ & $4,898,924$ & $1,537,996$ & $6,436,920$ \\
\hline
\end{tabular}


mouth, pharynx, and oesophagus, could be attributed to SLT in 2010. This is a little higher than the estimates from a recent study in which 50,000 deaths were attributed to SLT in eight South Asian countries [4]. This discrepancy may be explained by the fact that we used the most recent, updated prevalence and burden of disease figures.

Our estimate does not include economic impact. However, given the nature of the associated diseases, it is likely that the SLT use imposes a huge economic burden on weak health systems and poor economies. Moreover, owing to higher consumption of SLT among people of lower socio-economic status and inequitable access to health care in low-income and middle-income countries, its use is likely to contribute to driving disadvantaged sections of these societies into further poverty. A disproportionate impact on the male population (more than $70 \%$ of disease burden due to SLT is in males) is also likely to have a disproportionate economic impact on societies in terms of reduced workforce contributions by men. On the other hand, effective legislation, policy, and preventive programmes could avert this burden due to SLT.

The signatories of the WHO's Framework Convention on Tobacco Control should, in addition to the focus on reducing smoking consumption and related harm, now also consider the need to regulate production, marketing, and labelling of SLT products. This is particularly necessary in those countries where prevalence is high and SLT products are manufactured at a large scale without any checks on the carcinogenic level of their ingredients [35]. In countries where its use is largely limited to immigrant populations (such as in the UK) [36], strict regulation and taxation policies should be enforced which prevent import of SLT products and sale by local shops.

SLT is an important health issue, applying to a large part of the world. The data presented here are the most comprehensive gathered and brought together thus far. However, considerable uncertainties remain pertaining to risk estimation of different diseases associated with SLT use. Therefore more research is needed to investigate the newly established and previously known adverse health outcomes pertaining to SLT, particularly within countries where prevalence is high but no research evidence of risk estimation is available. Moreover, more descriptive questions about the type of SLT products and the pattern of use should be introduced into national surveys and publications of such findings encompassing all the regions.

\section{Conclusions}

Our study, a first attempt to assess global burden of disease due to SLT, estimates that more than six million
DALYs are lost and over a quarter of a million deaths occur each year owing to its consumption. There is a need to build on the insights obtained from efforts to reduce cigarette smoking-related harm and to investigate strategies to reduce use of SLT and decrease the substantial associated burden of harm.

\section{Additional file}

Additional file 1: Supplementary description of methods and results sections. (DOCX $281 \mathrm{~kb})$

\section{Abbreviations}

Cl: Confidence intervals; DALYs: Disability-adjusted life years; DHS: Demographic and Health Surveys; GATS: Global Adult Tobacco Survey; ICS: Individual Country Survey; PAF: Population attributable fraction; SEBS: Special Europe Barometer Survey; SLT: Smokeless tobacco; STEPS: STEPwise Approach to Surveillance; TSNA: Tobacco-specific nitrosamines; WHO: World Health Organization.

\section{Competing interests}

$\mathrm{KS}, \mathrm{SS}, \mathrm{SA}, \mathrm{AV}, \mathrm{MJ}$, and $\mathrm{OD}$ declare that they have no competing interests. AS reports grants from The Commonwealth Fund, outside the submitted work but no other conflict of interest.

\section{Authors' contributions}

KS: Developed the protocol, data analysis, data interpretation, drafting and approval of the manuscript. SS: Data collection, data analysis, data interpretation, contribution to and approval of the manuscript. SA: Co-drafted the protocol, data collection, data analysis, contribution to and approval of the manuscript. AV: Data collection, data analysis, data interpretation, contribution to and approval of the manuscript. MJ: Data collection, data interpretation, contribution to and approval of the manuscript. OD: Contribution to the protocol, data interpretation, contribution to and approval of the manuscript. AS: Conceived the idea, contribution to the protocol, data interpretation, contribution to and approval of the manuscript.

\section{Funding}

This study was funded by grants received from Leeds City Council, Leeds, UK and Medical Research Council, UK (MC_PC_13081).

\section{Ethics approval}

No ethics approval was required for this study.

\section{Author details}

${ }^{1}$ Department of Health Sciences, Hull York Medical School, University of York, Room 105a, First floor, ARRC Building, Heslington, York YO10 5DD, UK. ${ }^{2}$ Fatima Memorial Hospital College of Medicine and Dentistry, Fatima Memorial System, Shadman, Lahore 48000, Pakistan. ${ }^{3}$ Department of Primary Care and Public Health, Imperial College London, Charing Cross Campus, Reynold's Building, Hammersmith W6 8RP, UK. “Usher Institute of Population Health Sciences and Informatics, The University of Edinburgh, Medical School Doorway 3, Teviot Place, Edinburgh EH8 9AG, UK.

Received: 6 May 2015 Accepted: 17 July 2015

Published online: 17 August 2015

\section{References}

1. CDC. Smokeless Tobacco Fact Sheets. Prepared for the 3rd International Conference on Smokeless tobacco: advancing Science and Protecting Public Health. Stockholm, Sweden: National Cancer Institute, CDC and Prevention \& Stockholm Centre of Public Health; 2002.

2. SEARO. WHO: report on oral tobacco use and its implications in South East Asia. New Delhi: SEARO; 2004.

3. Palipudi K, Rizwan SA, Sinha DN, Andes LJ, Amarchand R, Krishnan A, et al. Prevalence and sociodemographic determinants of tobacco use in four countries of the World Health Organization: South-East Asia region: findings from the Global Adult Tobacco Survey. Indian J Cancer. 2014;51:S24-32. 
4. Boffetta P, Hecht S, Gray N, Gupta P, Straif K. Smokeless tobacco and cancer. Lancet Oncol. 2008;9:667-75.

5. International Agency for Research on Cancer. Smokeless tobacco products. In: IARC Monographs on the Evaluation of Carcinogenic Risks to Humans, vol. 89. Lyon, France: International Agency for Research on Cancer; 2008.

6. Stanfill SB, Connolly GN, Zhang L, Jia LT, Henningfield JE, Richter P, et al. Global surveillance of oral tobacco products: total nicotine, unionised nicotine and tobacco-specific N-nitrosamines. Tob Control. 2011;20:e2.

7. Malson $J$, Sims K, Murty R, Pickworth WB. Comparison of the nicotine content of tobacco used in bidis and conventional cigarettes. Tob Control. 2001;10:181-3.

8. Critchley JA, Unal B. Health effects associated with smokeless tobacco: a systematic review. Thorax. 2003;58:435-43.

9. Gupta PC, Ray CS, Sinha DN, Singh PK. Smokeless tobacco: a major public health problem in the SEA region: a review. Indian J Public Health. 2011;55:199-209.

10. Gupta PC, Sreevidya S. Smokeless tobacco use, birth weight, and gestational age: population based, prospective cohort study of 1217 women in Mumbai, India. BMJ. 2004;328:1538.

11. Boffetta P, Straif K. Use of smokeless tobacco and risk of myocardial infarction and stroke: systematic review with meta-analysis. BMJ. 2009;339:b3060.

12. Gupta PC, Subramoney S. Smokeless tobacco use and risk of stillbirth: a cohort study in Mumbai, India. Epidemiology. 2006;17:47-51.

13. England $\sqcup$, Kim SY, Shapiro-Mendoza CK, Wilson HG, Kendrick JS, Satten GA, et al. Maternal smokeless tobacco use in Alaska Native women and singleton infant birth size. Acta Obstet Gynecol Scand. 2012;91:93-103.

14. Tomar SL. Is use of smokeless tobacco a risk factor for cigarette smoking? The U.S. experience. Nicotine Tob Res. 2003;5:561-9.

15. Zhou J, Michaud DS, Langevin SM, McClean MD, Eliot M, Kelsey KT. Smokeless tobacco and risk of head and neck cancer: evidence from a case-control study in New England. Int J Cancer. 2013;132:1911-7.

16. Napier AD, Ancarno C, Butler B, Calabrese J, Chater A, Chatterjee H, et al. Culture and health. Lancet. 2014;384:1607-39.

17. Lawler TS, Stanfill SB, Zhang L, Ashley DL, Watson CH. Chemical characterization of domestic oral tobacco products: total nicotine, $\mathrm{pH}$, unprotonated nicotine and tobacco-specific N-nitrosamines. Food Chem Toxicol. 2013;57:380-6.

18. Ezzati M, Lopez AD, Rodgers A, Vander Hoorn S, Murray CJ. Selected major risk factors and global and regional burden of disease. Lancet. 2002:360:1347-60.

19. Lim SS, Vos T, Flaxman AD, Danaei G, Shibuya K, Adair-Rohani H, et al. A comparative risk assessment of burden of disease and injury attributable to 67 risk factors and risk factor clusters in 21 regions, 1990-2010: a systematic analysis for the Global Burden of Disease Study 2010. Lancet. 2012;380:2224-60.

20. Horton R, Chan M, Kim J, Watts C, Cairncross S. Global Burden of Disease Study 2010. Lancet. 2012;380:2053-260.

21. World Health Organization. The World Health Report 2002: reducing risks, promoting healthy life. Geneva: World Health Organization; 2002.

22. CDC. Global Tobacco Surveillance System Data. www.cdc.gov/tobacco/global/gtss/.

23. The Newcastle-Ottawa Scale (NOS) for assessing the quality of nonrandomised studies in meta-analyses [http://www.ohri.ca/programs/ clinical_epidemiology/oxford.asp].

24. Chinn S. A simple method for converting an odds ratio to effect size for use in meta-analysis. Stat Med. 2000;19:3127-31.

25. Rockhill B, Newman B, Weinberg C. Use and misuse of population attributable fractions. Am J Public Health. 1998;88:15-9.

26. Öberg M, Jaakkola MS, Woodward A, Peruga A, Prüss-Ustün A. Worldwide burden of disease from exposure to second-hand smoke: a retrospective analysis of data from 192 countries. Lancet. 2011;377:139-46.

27. Murray CJ, Vos T, Lozano R, Naghavi M, Flaxman AD, Michaud C, et al. Disability-adjusted life years (DALYs) for 291 diseases and injuries in 21 regions, 1990-2010: a systematic analysis for the Global Burden of Disease Study 2010. Lancet. 2012;380:2197-223.

28. Lozano R, Naghavi M, Foreman K, Lim S, Shibuya K, Aboyans V, et al. Global and regional mortality from 235 causes of death for 20 age groups in 1990 and 2010: a systematic analysis for the Global Burden of Disease Study 2010. Lancet. 2012:380:2095-128.

29. Teo KK, Ounpuu S, Hawken S, Pandey MR, Valentin V, Hunt D, et al. Tobacco use and risk of myocardial infarction in 52 countries in the INTERHEART study: a case-control study. Lancet. 2006;368:647-58.

30. Idris AM, Ibrahim Y, Warnakulasuriya K, Cooper D, Johnson N, Nilsen R. Toombak use and cigarette smoking in the Sudan: estimates of prevalence in the Nile state. Prev Med. 1998;27:597-603.
31. Siddiqi K, Gupta PC, Prasad VM, Croucher R, Sheikh A. Smokeless tobacco use by south Asians. Lancet Glob Health. 2013;1:e71.

32. Sinha DN, Palipudi KM, Jones CK, Khadka BB, Silva PD, Mumthaz M, et al. Levels and trends of smokeless tobacco use among youth in countries of the World Health Organization South-East Asia Region. Indian J Cancer. 2014;51:S50-53.

33. Norberg M, Malmberg G, Ng N, Broström G. Who is using snus? - Time trends, socioeconomic and geographic characteristics of snus users in the ageing Swedish population. BMC Public Health. 2011;11:929-9.

34. Rodu B, Stegmayr B, Nasic S, Cole P, Asplund K. Evolving patterns of tobacco use in northern Sweden. J Intern Med. 2003;253:660-5.

35. Khan A, Huque R, Shah SK, Kaur J, Baral S, Gupta PC, et al. Smokeless tobacco control policies in South Asia: a gap analysis and recommendations. Nicotine Tob Res. 2014;16:890-4.

36. Panesar SS, Gatrad R, Sheikh A. Smokeless tobacco use by south Asian youth in the UK. Lancet. 2008;372:97-8.

37. National Cancer Institute and Centers for Disease Control and Prevention. Smokeless tobacco and public health: a global perspective. Bethesda, MD: US Department of Health and Human Services, Centres for Disease Control and Prevention, and National Institute of Health, National Cancer Institute; 2014.

38. STEPS Country Reports [http://www.who.int/chp/steps/reports/en/].

39. Sreeramareddy CT, Pradhan P, Sin S. Prevalence, distribution, and social determinants of tobacco use in 30 sub-Saharan African countries. BMC Med. 2014;12:243

40. Ansra DL, Arnold F, Kishor S, Hsia J, Kaufmann R. Tobacco use by men and women in 49 countries with demographic and health surveys. DHS Comparative Reports No 31. Calverton, MD: ICF International; 2013.

41. World Health Organization. WHO report on the global tobacco epidemic, 2013: enforcing bans on tobacco advertising, promotion and sponsorship. Geneva: WHO; 2013.

42. GATS (Global Adult Tobacco Survey) [http://www.who.int/tobacco/ surveillance/gats/en/].

43. Sreeramareddy CT, Pradhan PM, Mir IA, Sin S. Smoking and smokeless tobacco use in nine South and Southeast Asian countries: prevalence estimates and social determinants from Demographic and Health Surveys. Popul Health Metr. 2014;12:22.

44. Agaku IT, Filippidis FT, Vardavas Cl, Odukoya OO, Awopegba AJ, Ayo-Yusuf OA, et al. Poly-tobacco use among adults in 44 countries during 2008-2012: evidence for an integrative and comprehensive approach in tobacco control. Drug Alcohol Depend. 2014;139:60-70.

45. Australian Institute of Health and Welfare. 2004 National Drug Strategy Household Survey: first results. Canberra: Australian Institute of Health and Welfare; 2005.

46. Anantharaman D, Chaubal PM, Kannan S, Bhisey RA, Mahimkar MB. Susceptibility to oral cancer by genetic polymorphisms at CYP1A1, GSTM1 and GSTT1 loci among Indians: tobacco exposure as a risk modulator. Carcinogenesis. 2007;28:1455-62.

47. Balaram P, Sridhar H, Rajkumar T, Vaccarella S, Herrero R, Nandakumar A et al. Oral cancer in southern India: the influence of smoking, drinking, paan-chewing and oral hygiene. Int J Cancer. 2002;98:440-5.

48. Dikshit RP, Kanhere S. Tobacco habits and risk of lung, oropharyngeal and oral cavity cancer: a population-based case-control study in Bhopal, India. Int J Epidemiol. 2000;29:609-14.

49. Goud ML, Mohapatra SC, Mohapatra P, Gaur SD, Pant GC, Knanna MN Epidemiological correlates between consumption of Indian chewing tobacco and oral cancer. Eur J Epidemiol. 1990;6:219-22.

50. Jayalekshmi PA, Gangadharan P, Akiba S, Nair RRK, Tsuji M, Rajan B. Tobacco chewing and female oral cavity cancer risk in Karunagappally cohort, India. Br J Cancer. 2009;100:848-52.

51. Jayalekshmi PA, Gangadharan P, Akiba S, Koriyama C, Nair RRK. Oral cavity cancer risk in relation to tobacco chewing and bidi smoking among men in Karunagappally, Kerala, India: Karunagappally cohort study. Cancer Sci. 2011;102:460-7.

52. Jayant K, Balakrishnan V, Sanghvi LD, Jussawalla DJ. Quantification of the role of smoking and chewing tobacco in oral, pharyngeal, and oesophageal cancers. Br J Cancer. 1977;35:232-5.

53. Jussawalla DJ, Deshpande VA. Evaluation of cancer risk in tobacco chewers and smokers: an epidemiologic assessment. Cancer. 1971;28:244-52.

54. Madani AH, Jahromi AS, Dikshit M, Bhaduri D. Risk assessment of tobacco types and oral cancer. Am J Pharmacol Toxicol. 2010;5:9-13.

55. Muwonge R, Ramadas K, Sankila R, Thara S, Thomas G, Vinoda J, et al. Rote of tobacco smoking, chewing and alcohol drinking in the risk of oral cancer 
in Trivandrum, India: A nested case-control design using incident cancer cases. Oral Oncol. 2008;44:446-54.

56. Nandakumar A, Thimmasetty KT, Sreeramareddy NM, Venugopal TC, Rajanna, Vinutha AT, et al. A population-based case control investigation on cancers of the oral cavity in Bangalore, India. British Journal of Cancer. 1990;62:847-51.

57. Rao DN, Ganesh B, Rao RS, Desai PB. Risk assessment of tobacco, alcohol and diet in oral cancer: a case-control study. Int J Cancer. 1994;58:469-73.

58. Sanghvi LD, Rao KC, Khanolkar VR. Smoking and chewing of tobacco in relation to cancer of the upper alimentary tract. Br Med J. 1955:1:1111-4.

59. Sankaranarayanan R, Duffy SW, Padmakumary G, Day NE, Nair MK. Risk factors for cancer of the buccal and labial mucosa in Kerala, southern India. J Epidemiol Community Health. 1990;44:286-92.

60. Wasnik KS, Ughade SN, Zodpey SP, Ingole DL. Tobacco consumption practices and risk of oro-pharyngeal cancer: a case-control study in Central India. Southeast Asian J Trop Med Publ Health. 1998;29:827-34.

61. Subapriya R, Thangavelu A, Mathavan B, Ramachandran CR, Nagini S Assessment of risk factors for oral squamous cell carcinoma in Chidambaram, Southern India: a case-control study. Eur J Cancer Prev. 2007; 16:251-6.

62. Wahi PN, Kehar U, Lahiri B. Factors influencing oral and oropharyngeal cancers in India. Br J Cancer. 1965;19:642-60.

63. Merchant A, Husain SS, Hosain M, Fikree FF, Pitiphat W, Siddiqui AR, et al Paan without tobacco: an independent risk factor for oral cancer. Int J Cancer. 2000;86:128-31.

64. Roosaar A, Johansson AL, Sandborgh-Englund G, Axell T, Nyren O. Cancer and mortality among users and nonusers of snus. Int J Cancer. 2008;123:168-73.

65. Znaori A, Brennan P, Gajalakshmi V, Mathew A, Shanta V, Varghese C, et al. Independent and combined effects of tobacco smoking, chewing and alcohol drinking on the risk of oral, pharyngeal and esophageal cancers in Indian men. Int J Cancer. 2003;105:681-6.

66. Boffetta $P$, Aagnes B, Weiderpass E, Andersen A. Smokeless tobacco use and risk of cancer of the pancreas and other organs. Int J Cancer. 2005;114:992-5

67. Lewin F, Norell SE, Johansson H, Gustavsson P, Wennerberg J, Biörklund A, et al. Smoking tobacco, oral snuff, and alcohol in the etiology of squamous cell carcinoma of the head and neck: a population-based case-referent study in Sweden. Cancer. 1998:82:1367-75.

68. Luo J, Ye W, Zendehdel K, Adami J, Adami H-O, Boffetta $P$, et al. Oral use of Swedish moist snuff (snus) and risk for cancer of the mouth, lung, and pancreas in male construction workers: a retrospective cohort study. Lancet. 2007;369:2015-20

69. Rosenquist K, Wennerberg J, Schildt EB, Bladstrom A, Hansson BG, Andersson G. Use of Swedish moist snuff, smoking and alcohol consumption in the aetiology of oral and oropharyngeal squamous cell carcinoma. A population-based case-control study in southern Sweden. Acta Otolaryngol. 2005;125:991-8.

70. Schildt EB, Eriksson M, Hardell L, Magnuson A. Oral snuff, smoking habits and alcohol consumption in relation to oral cancer in a Swedish casecontrol study. Int J Cancer. 1998;77:341-6.

71. Mashberg A, Boffetta P, Winkelman R, Garfinkel L. Tobacco smoking, alcohol drinking, and cancer of the oral cavity and oropharynx among U.S. veterans. Cancer. 1993;72:1369-75.

72. Sapkota A, Gajalakshmi V, Jetly DH, Roychowdhury S, Dikshit RP, Brennan P, et al. Smokeless tobacco and increased risk of hypopharyngeal and laryngeal cancers: A multicentric case-control study from India. Int J Cancer. 2007:121:1793-8.

73. Akhtar S, Sheikh AA, Qureshi HU. Chewing areca nut, betel quid, oral snuff, cigarette smoking and the risk of oesophageal squamous-cell carcinoma in South Asians: a multicentre case-control study. Eur J Cancer. 2012:48:655-61.

74. Dar NA, Bhat GA, Shah IA, lqbal B, Makhdoomi MA, Nisar I, et al. Hookah smoking, nass chewing, and oesophageal squamous cell carcinoma in Kashmir, India. [Erratum appears in Br J Cancer. 2013;108(7):1552 Note: Kakhdoomi MA [corrected to Makhdoomi MA]]. Br J Cancer. 2012;107:1618-23.

75. Sehgal S, Kaul S, Gupta BB, Dhar MK. Risk factors and survival analysis of the esophageal cancer in the population of Jammu, India. Indian J Cancer. 2012:49:245-50.
76. Talukdar FR, Ghosh SK, Laskar RS, Mondal R. Epigenetic, genetic and environmental interactions in esophageal squamous cell carcinoma from northeast India. PLoS One. 2013;8:e60996.

77. Lagergren J, Bergstrom R, Lindgren A, Nyren O. The role of tobacco, snuff and alcohol use in the aetiology of cancer of the oesophagus and gastric cardia. Int J Cancer. 2000;85:340-6.

78. Zendehdel K, Nyren O, Luo J, Dickman PW, Boffetta P, Englund A, et al. Risk of gastroesophageal cancer among smokers and users of Scandinavian moist snuff. Int J Cancer. 2008;122:1095-9.

79. Bolinder G, Alfredsson L, Englund A, de Faire U. Smokeless tobacco use and increased cardiovascular mortality among Swedish construction workers. Am J Public Health. 1994;84:399-404.

80. Sasco AJ, Merrill RM, Dari I, Benhaim-Luzon V, Carriot F, Cann Cl, et al. A case-control study of lung cancer in Casablanca, Morocco. Cancer Causes Control. 2002;13:609-16.

81. Brown LM, Blot WJ, Schuman SH, Smith VM, Ershow AG, Marks RD, et al. Environmental factors and high risk of esophageal cancer among men in coastal South Carolina. J Natl Cancer Inst. 1988:80:1620-5.

82. Alguacil J, Silverman DT. Smokeless and other noncigarette tobacco use and pancreatic cancer: a case-control study based on direct interviews. Cancer Epidemiol Biomarkers Prev. 2004;13:55-8.

83. Hassan MM, Abbruzzese JL, Bondy ML, Wolff RA, Vauthey JN, Pisters PW et al. Passive smoking and the use of noncigarette tobacco products in association with risk for pancreatic cancer: a case-control study. Cancer. 2007;109:2547-56.

84. Alexander M. Tobacco use and the risk of cardiovascular diseases in developed and developing countries. Cambridge: University of Cambridge; 2013.

85. Rahman MA, Zaman MM. Smoking and smokeless tobacco consumption: possible risk factors for coronary heart disease among young patients attending a tertiary care cardiac hospital in Bangladesh. Public Health. 2008;122:1331-8.

86. Rahman MA, Spurrier N, Mahmood MA, Rahman M, Choudhury SR, Leeder S. Is there any association between use of smokeless tobacco products and coronary heart disease in Bangladesh? PLoS One. 2012;7:e30584.

87. Hergens MP, Ahlbom A, Andersson T, Pershagen G. Swedish moist snuff and myocardial infarction among men. Epidemiology. 2005;16:12-6.

88. Hergens MP, Alfredsson L, Bolinder G, Lambe M, Pershagen G, Ye W. Long-term use of Swedish moist snuff and the risk of myocardial infarction amongst men. [Erratum appears in J Intern Med. 2007;262(5):590]. J Intern Med. 2007;262:351-9.

89. Huhtasaari F, Asplund K, Lundberg V, Stegmayr B, Wester PO. Tobacco and myocardial infarction: is snuff less dangerous than cigarettes? BMJ. 1992;305:1252-6.

90. Huhtasaari F, Lundberg V, Eliasson M, Janlert U, Asplund K. Smokeless tobacco as a possible risk factor for myocardial infarction: a populationbased study in middle-aged men. J Am Coll Cardiol. 1999;34:1784-90.

91. Johansson S-E, Sundquist K, Qvist J, Sundquist J. Smokeless tobacco and coronary heart disease: a 12-year follow-up study. [Erratum appears in Eur J Cardiovasc Prev Rehabil. 2007;14(5):722]. Eur J Cardiovasc Prev Rehabil. 2005;12:387-92.

92. Wennberg P, Eliasson M, Hallmans G, Johansson L, Boman K, Jansson JH. The risk of myocardial infarction and sudden cardiac death amongst snuff users with or without a previous history of smoking. J Intern Med. 2007;262:360-7.

93. Asplund K, Nasic S, Janlert U, Stegmayr B. Smokeless tobacco as a possible risk factor for stroke in men - a nested case-control study. Stroke. 2003;34:1754-9.

94. Hergens M-P, Lambe M, Pershagen G, Terent A, Ye W. Smokeless tobacco and the risk of stroke.[Erratum appears in Epidemiology. 2009;20(3):471]. Epidemiology. 2008;19:794-9.

95. Hansson J, Pedersen NL, Galanti MR, Andersson T, Ahlbom A, Hallqvist J, et al. Use of snus and risk for cardiovascular disease: results from the Swedish Twin Registry. J Intern Med. 2009;265:717-24.

96. Janzon E, Hedblad B. Swedish snuff and incidence of cardiovascular disease. A population-based cohort study. BMC Cardiovasc Disord. 2009;9:21. 